\title{
RBMS1 regulates lung cancer ferroptosis through translational control of SLC7A11
}

\author{
Wenjing Zhang, ${ }^{1}$ Yu Sun, ${ }^{1}$ Lu Bai, ${ }^{1}$ Lili Zhi, ${ }^{1}$ Yun Yang, ${ }^{2}$ Qingzhi Zhao, ${ }^{1}$ Chaoqun Chen, ${ }^{1}$ Yangfan Qi, ${ }^{1}$ Wenting Gao, ${ }^{3}$ Wenxia He, ${ }^{1}$ \\ Luning Wang, ${ }^{1}$ Dan Chen, ${ }^{4}$ Shujun Fan, ${ }^{5}$ Huan Chen, ${ }^{6}$ Hai-Long Piao, ${ }^{6}$ Qinglong Qiao, ${ }^{6}$ Zhaochao Xu, ${ }^{6}$ Jinrui Zhang, ${ }^{1}$ Jinyao Zhao, ${ }^{1}$ \\ Sirui Zhang, ${ }^{2}$ Yue Yin, ${ }^{7}$ Chao Peng, ${ }^{7}$ Xiaoling Li, ${ }^{8}$ Quentin Liu, ${ }^{1}$ Han Liu, ${ }^{1}$ and Yang Wang ${ }^{1}$ \\ Institute of Cancer Stem Cells and Second Affiliated Hospital, Dalian Medical University, Dalian, China. ${ }^{2}$ CAS-MPG Partner Institute for Computational Biology, Shanghai Institute of Nutrition and Health, \\ Chinese Academy of Sciences, Shanghai, China. ${ }^{3}$ nstitute of Cenome Engineered Animal Models for Human Diseases, ${ }^{4}$ Department of Pathology, First Affiliated Hospital, and ${ }^{5}$ Department of Pathology, \\ Dalian Medical University, Dalian, China. ${ }^{6}$ CAS Key Laboratory of Separation Science for Analytical Chemistry, Dalian Institute of Chemical Physics, Chinese Academy of Sciences, Dalian, China. ${ }^{7}$ National \\ Facility for Protein Science in Shanghai, Zhangjiang Lab, Shanghai Advanced Research Institute, Chinese Academy of Science, Shanghai, China. ${ }^{8}$ Signal Transduction Laboratory, National Institute of \\ Environmental Health Sciences, Research Triangle Park, North Carolina, USA.
}

Ferroptosis, an iron-dependent nonapoptotic cell death, is a highly regulated tumor suppressing process. However, functions and mechanisms of RNA-binding proteins in regulation of evasion of ferroptosis during lung cancer progression are still largely unknown. Here, we report that the RNA-binding protein RBMS1 participates in lung cancer development via mediating ferroptosis evasion. Through an shRNA-mediated systematic screen, we discovered that RBMS1 is a key ferroptosis regulator. Clinically, RBMS1 was elevated in lung cancer and its high expression was associated with reduced patient survival. Conversely, depletion of RBMS1 inhibited lung cancer progression both in vivo and in vitro. Mechanistically, RBMS1 interacted with the translation initiation factor elF3d directly to bridge the $3^{\prime}$ - and 5'-UTR of SLC7A11. RBMS1 ablation inhibited the translation of SLC7A11, reduced SLC7A11-mediated cystine uptake, and promoted ferroptosis. In a drug screen that targeted RBMS1, we further uncovered that nortriptyline hydrochloride decreased the level of RBMS1, thereby promoting ferroptosis. Importantly, RBMS1 depletion or inhibition by nortriptyline hydrochloride sensitized radioresistant lung cancer cells to radiotherapy. Our findings established RBMS1 as a translational regulator of ferroptosis and a prognostic factor with therapeutic potential and clinical value.

\section{Introduction}

Ferroptosis, an iron-dependent form of nonapoptotic cell death, is induced by cystine depletion and massive lipid peroxidation-controlled membrane damage (1-4). Dysregulation of ferroptosis has been closely associated with human cancers $(5$, 6 ). Accumulating evidence has demonstrated that ferroptosis plays a vital role in tumor suppression (7-9). Traditional chemotherapeutic drugs (e.g., cisplatin) combined with ferroptosis inducers can synergistically inhibit tumor proliferation in head and neck cancer (10). Activation of ferroptosis is able to prevent acquired resistance of cancer cells to several cancer therapies, including lapatinib, erlotinib, and vemurafenib (11-13). Moreover, immunotherapy with anti-PD-L1 antibodies stimulates lipid peroxidation-dependent ferroptosis in tumor cells; ferroptosis and immunotherapy can therefore synergistically inhibit tumor growth in vitro and in vivo (14). Importantly, ferroptosis can also be induced by irradiation and is as important as apoptosis in irradiation-induced tumor suppression $(15,16)$, imply-

Authorship note: WZ, YS, LB, LZ, and Y Yang contributed equally to this work Conflict of interest: The authors have declared that no conflict of interest exists. Copyright: (5) 2021, American Society for Clinical Investigation.

Submitted: June 4, 2021; Accepted: September 28, 2021; Published: November 15, 2021. Reference information: / Clin Invest. 2021;131(22):e152067.

https://doi.org/10.1172/JCl152067. ing that activation of ferroptosis might sensitize radioresistant cancer cells to radiotherapy. Therefore, targeting ferroptosis provides great potential for cancer therapy.

Ferroptosis can be regulated by multiple critical factors, including solute carrier family 7 member 11 (SLC7A11, the key transporter of cystine), and glutathione peroxidase 4 (GPX4) (1720). SLC7A11 imports extracellular cystine into the cell (21), which is subsequently converted to glutathione (GSH). GPX4 is capable of using GSH to decrease lipid hydroperoxides, thereby inhibiting ferroptosis (17). Thus, SLC7A11 and GPX4 are suppressors of ferroptosis, and inhibition of SLC7A11 or GPX4 with distinct approaches is able to trigger ferroptosis $(1,17)$. The expression and activity of SLC7A11 are regulated at multiple levels. Under oxidative stress, the transcription factor nuclear factor erythroid 2-related factor 2 (NRF2) binds to the antioxidant response elements (AREs) in the promotor region of $S L C 7 A 11$ to stimulate its transcription (22). The transcription of SCL7A11 can also be regulated by BAP1 and the methylation of histone $\mathrm{H} 3(7,23)$. Apart from transcriptional regulation, the level of SLC7A11 is also controlled by posttranslational regulation. For instance, OTUB1, a noncanonical deubiquitinase, interacts with SLC7A11 to prevent its degradation (24). The adhesion molecule CD44 variant $(\mathrm{CD} 44 \mathrm{v})$ serves as a binding partner of SLC7A11 to modulate its protein stability (25). However, despite these observations, translational regulators of ferroptosis during lung cancer progression are still largely unknown. 
A
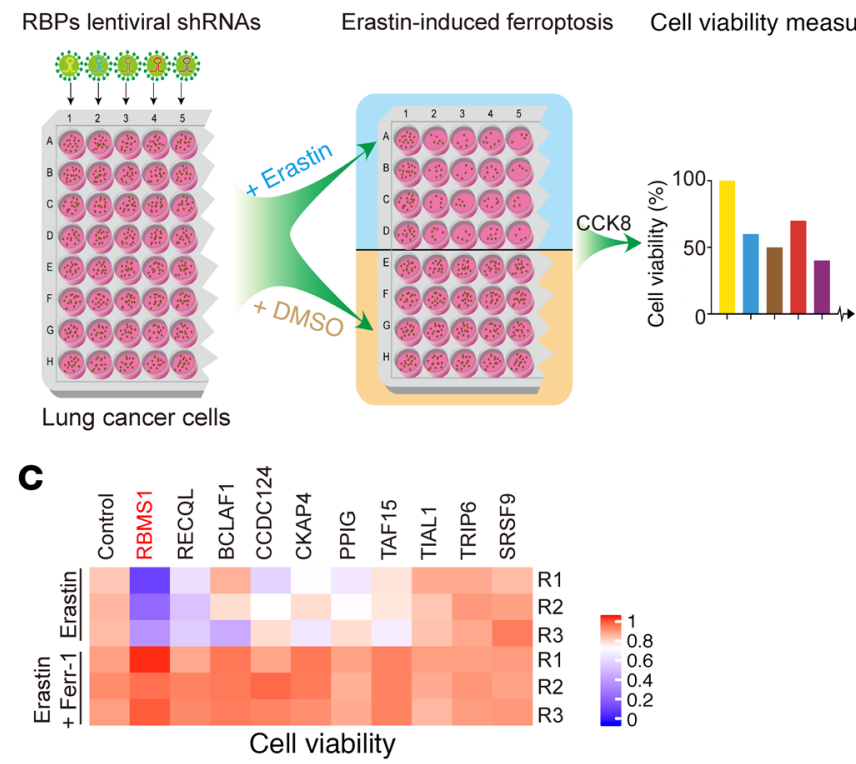

D

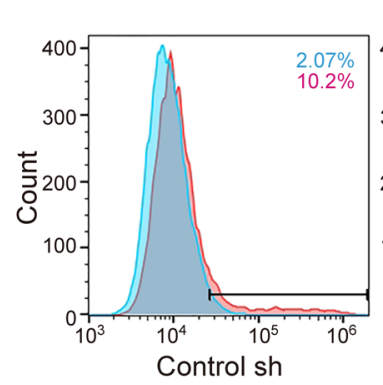

$\mathbf{F}$
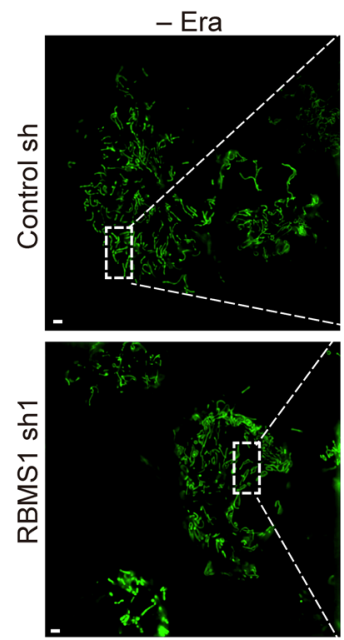

Lipid ROS level (FITC)
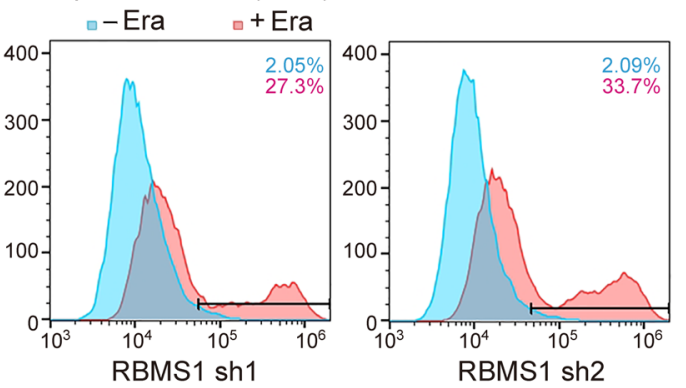

+ Era
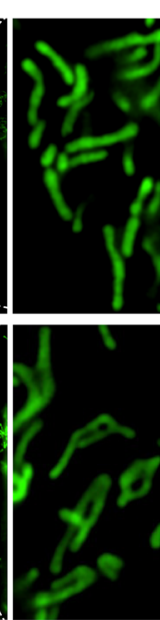

B

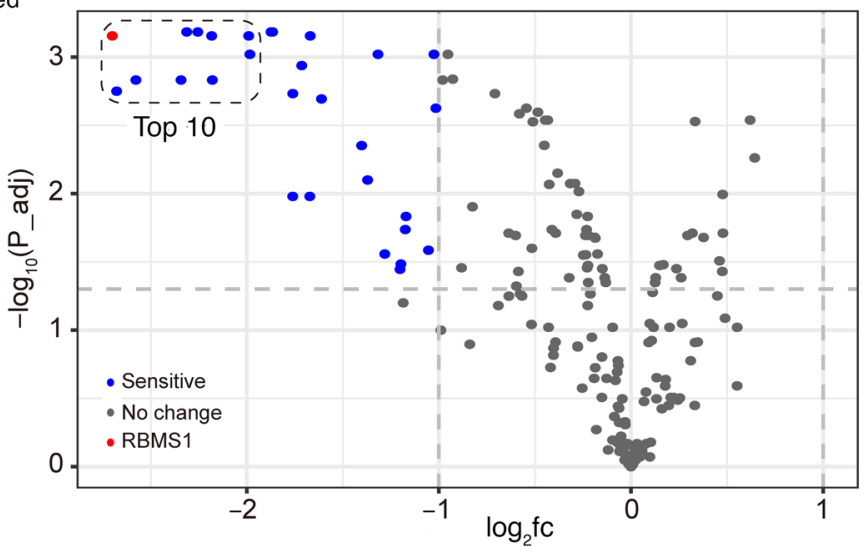

E
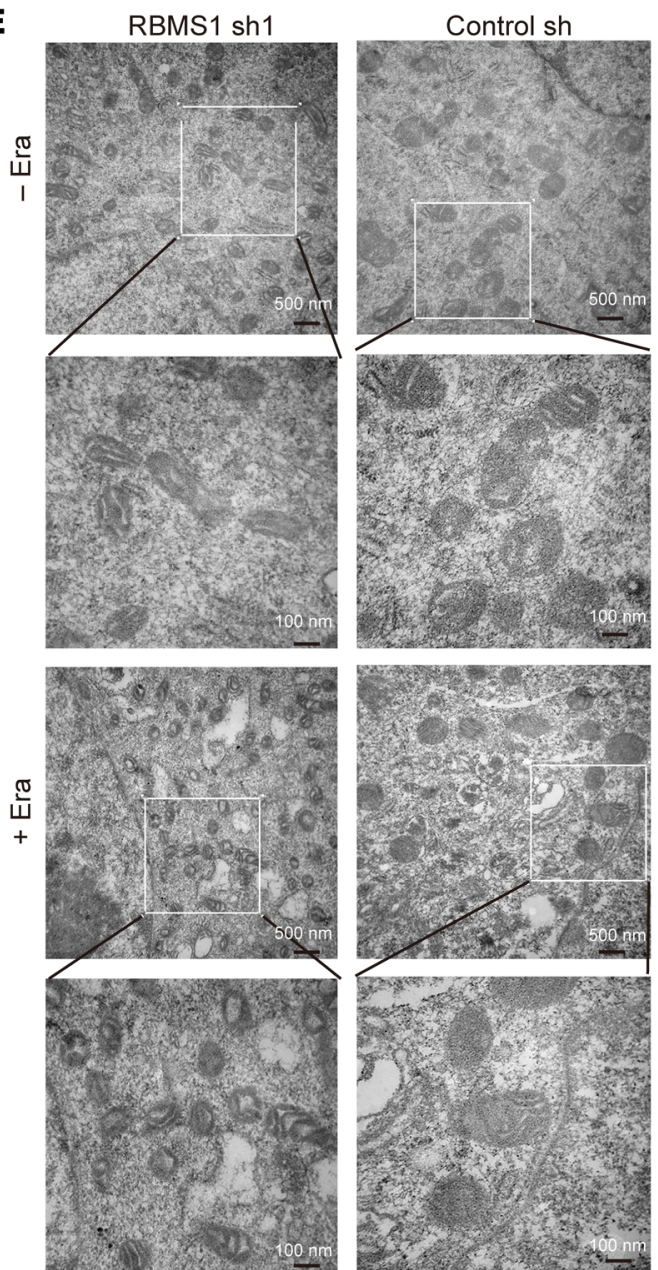

Figure 1. Systematic identification of RBMS1 as a key regulator of ferroptosis in lung cancer. (A) Workflow for RBP identification in ferroptosis regulation. A549 cells were infected with 190 viruses expressing shRNAs against distinct RBPs. (B) Volcano plot illustrating RBPs involved in regulating ferroptosis. The top 10 candidates are marked. (C) Heatmap depicting viability of A549 cells (with the top 10 RBPs identified in B depleted using distinct shRNAs) treated with erastin or erastin and ferrostatin-1 (Ferr-1). (D) Lipid peroxidation was measured by flow cytometry after C11-BODIPY staining in RBMS1-depleted H1299 cells. (E) RBMS1-depleted H1299 cells were treated with erastin and analyzed by TEM. Scale bars: 500 nm (rows 1 and 3) and 100 nm (rows 2 and 4). (F) RBMS1-depleted H1299 cells were treated with or without erastin and stained with Rho123, a known mitochondria-tracking probe, and analyzed by SIM. Scale bars: $2 \mu \mathrm{m}$. 
RNA-binding proteins (RBPs) are a diverse group of proteins that interact with RNAs to form ribonucleoprotein complexes, regulating the fate of their target RNAs and controlling various aspects of gene expression, such as RNA stability, pre-mRNA splicing, translation, and so on $(26,27,28)$. Importantly, RBPs are involved in the regulation of a variety of cancer-related biological processes, including apoptosis, epithelial-mesenchymal transition, as well as autophagy (29-31). However, the role of RBPs in regulating ferroptosis in lung cancer is still elusive. The RBP RBMS1 (also known as MSSP-1) was originally identified as a c-Myc gene single-stranded binding protein that could suppress transcription of target genes (32). Recently, RBMS1 was found to directly bind its target mRNAs to modulate RNA stability, thereby inhibiting colon cancer metastasis (33). However, the involvement of RBMS1 in lung cancer through translational regulation of ferroptosis-related genes has not yet been reported. Here, we systematically identified RBMS1 as a translation-promoting factor for SLC7A11 in lung cancer, and revealed that depletion of this RBP stimulates ferroptosis, thus inhibiting lung cancer cell growth and sensitizing lung cancer cells to radiotherapy.

\section{Results}

Systematic identification of RBMS1 as a key regulator of ferroptosis in lung cancer. To systematically identify RBPs that could regulate ferroptosis in lung cancer cells, we carried out an RNAi screen with an shRNA library targeting approximately 200 RBPs. The efficacy of this library has been previously validated by the ENCODE project (34). We induced ferroptosis using erastin in the RBP-depleted lung cancer cells, and then measured cell viability in response to ferroptosis induction (Figure 1A). We found that multiple RBPs could participate in ferroptosis regulation, and subsequently chose the top 10 candidate RBPs for further validation (Figure 1B). RBMS1 was identified as the top hit that substantially influenced ferroptosis (Figure 1B). RBMS1-depleted A549 lung cancer cells were extremely sensitive to erastin-induced ferroptosis, and this sensitivity was prevented by the ferroptosis inhibitor ferrostatin-1 (Ferr-1) (Figure 1C and Supplemental Figure 1A; supplemental material available online with this article; https://oi.org/10.1172/ JCI152067DS1), suggesting that depletion of RBMS1 promotes ferroptosis in lung cancer cells.

To further investigate the potential role of RBMS1 in ferroptosis, we generated stably RBMS1-depleted H1299 lung cancer cells with 2 independent shRNAs and found that knockdown of RBMS1 notably elevated erastin-triggered lipid peroxidation (Figure 1D and Supplemental Figure 1B). Consistently, CRISPR-mediated deletion of RBMS1 also increased erastin-induced lipid peroxidation in H1299 lung cancer cells (Supplemental Figure 1C). Transmission electron microscopy (TEM) analysis further revealed that RBMS1-depleted lung cancer cells contained shrunken mitochondria with elevated membrane density, a typical morphologic feature of ferroptosis (Figure 1E). Similar results were also observed in RBMS1-depleted H1299 cells stained with a known mitochondria-tracker probe, rhodamine 123 (Rho123), using a structured illumination microscopy (SIM) approach (Figure 1F).

Functionally, reduction in RBMS1 significantly promoted erastin-induced cell death (Figure 2A), which could be prevented by Ferr-1, in different lung cancer cells (Figure 2B and Supplemen- tal Figure 1D). As controls, inhibitors of other forms of cell death, including apoptosis (Z-VAD-FMK) and autophagy (3-methylademine, 3-MA) failed to suppress the erastin-triggered increase in cell death in distinct, stably RBMS1-depleted lung cancer cells (H1299 and A549 cells; Figure 2, C and D, and Supplemental Figure $1 \mathrm{E})$, indicating that the erastin-triggered cell death was independent of apoptosis and autophagy in lung cancer cells with stable depletion of RBMS1. Knocking out RBMS1 in lung cancer cells led to similar results (Supplemental Figure 2, A-E).

To further confirm that RBMS1 deficiency sensitizes lung cancer cells to ferroptosis, we induced ferroptosis with tert-butyl hydroperoxide (TBH), an ROS inducer, in control and RBMS1-knockout lung cancer cells. Deletion of RBMS1 in H1299 cells significantly increased cell death, which could also be substantially suppressed by the ferroptosis inhibitor Ferr-1 but not by the apoptosis inhibitor Z-VAD-FMK or the autophagy inhibitor 3-MA (Figure 2E and Supplemental Figure 3, A and B). Furthermore, knockdown of RBMS1 also sensitized H1299 cells to low-cystine-medium-induced ferroptosis, and this sensitivity was fully rescued by Ferr-1 (Figure 2, F and G). Subsequent reexpression of RBMS1 in H1299 cells with stable RBMS1 depletion markedly reversed the erastin-induced ferroptosis and significantly reduced cell death (Figure 2, H and I, and Supplemental Figure 3C). Altogether, our results demonstrated that loss of RBMS1 stimulates ferroptosis in lung cancer cells.

RBMS1 is associated with lung cancer progression and prognosis in humans and mice. Our identification of RBMS1 as a key regulator of ferroptosis in lung cancer cells raises the possibility that RBMS1 may participate in lung cancer progression by regulating ferroptosis evasion. To test this possibility, we sought to directly evaluate its clinical importance in lung cancer patients. We examined the expression of RBMS1 by immunohistochemistry (IHC) using serial sections of tissue microarrays containing lung cancer tissues $(n=$ 60 ) and the matched adjacent lung tissues ( $n=60$; Figure $3 \mathrm{~A})$. Specifically, more than $50 \%$ of lung cancer samples exhibited strong or extra-strong staining for RBMS1, whereas only less than $5 \%$ of adjacent lung tissues displayed strong or extra-strong staining for RBMS1 (Figure 3B). Moreover, lung cancer patients with a higher expression level of RBMS1 usually had a higher clinical stage tumor (Supplemental Figure 4A), indicating that the expression level of RBMS1 was positively correlated with the clinical stage of lung cancer patients. Furthermore, lung cancer patients with relatively high levels of RBMS1 had shorter overall survival than those with low levels of RBMS1 (Figure 3C). Therefore, our clinical observations indicate that RBMS1 is a potential prognostic factor in lung cancer.

We next investigated the effect of RBMS1 on lung cancer progression in mice using a conditional RBMS1 knockout (CKO) strain in which the homozygous floxed Rbms1 allele (Rbms $1^{f / f t}$ ) was crossed into the LSL-Kras ${ }^{G 12 D / W T}$ background (Kras ${ }^{G 12 D / W T}$ / $R b m s^{1 / f f l}$ ), with $\operatorname{Kras}^{G 12 D / W T} / R b m s 1^{W T}$ as controls (Supplemental Figure 4, B and C). Adeno-associated virus expressing Cre recombinase-GFP (AAV-Cre-GFP) was introduced into Kras ${ }^{G 12 D / W T} / R_{b m s} 1^{\text {WT }}$ and $\mathrm{Kras}^{G 12 \mathrm{D} / W T} / \mathrm{Rbms}^{\text {fl/fl }} \mathrm{CKO}$ mice to induce the knockout of RBMS1 and expression of $\mathrm{Kras}^{\mathrm{G} 12 \mathrm{D}}$ protein. The equal introduction of GFP-Cre signals was confirmed in lungs by in vivo fluorescence imaging (Supplemental Figure 4D). Strikingly, when imaged at 3, 5, and 8 weeks after AAV-Cre infection with coronal and transverse 
A
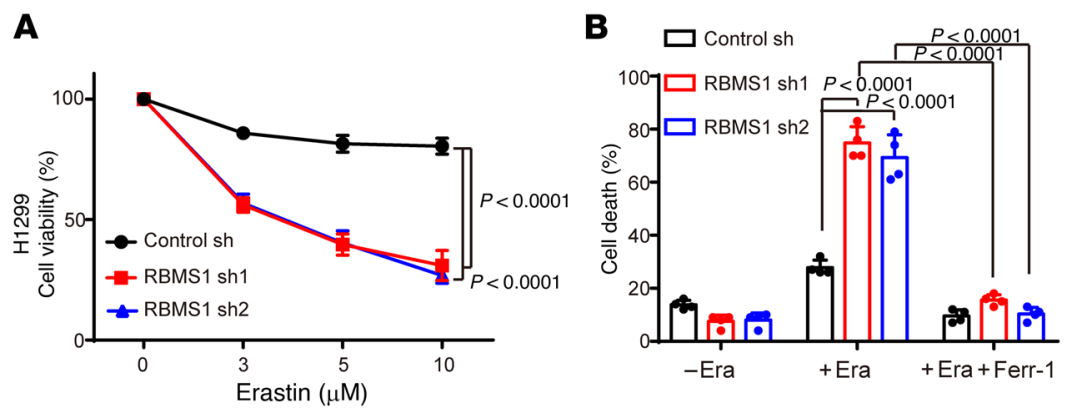

D

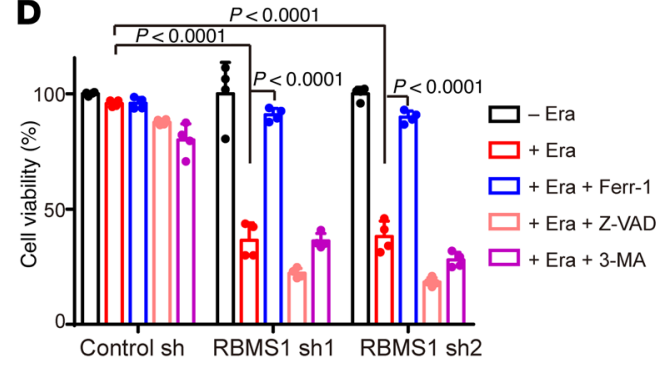

C
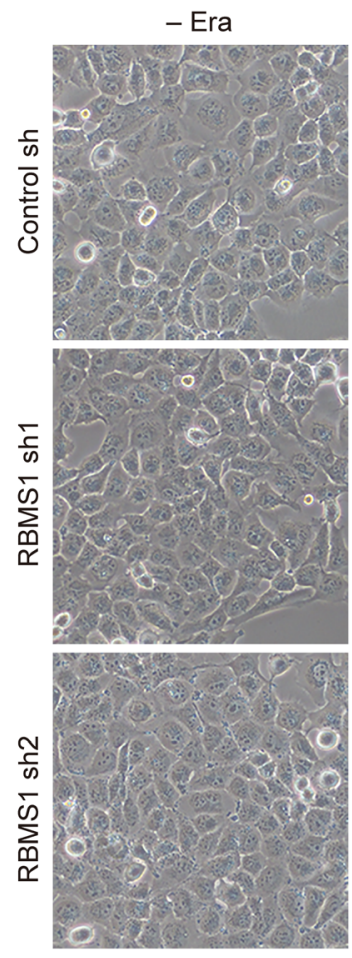

E

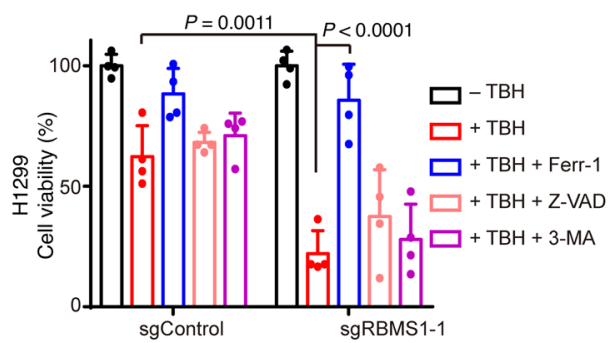

+ Era
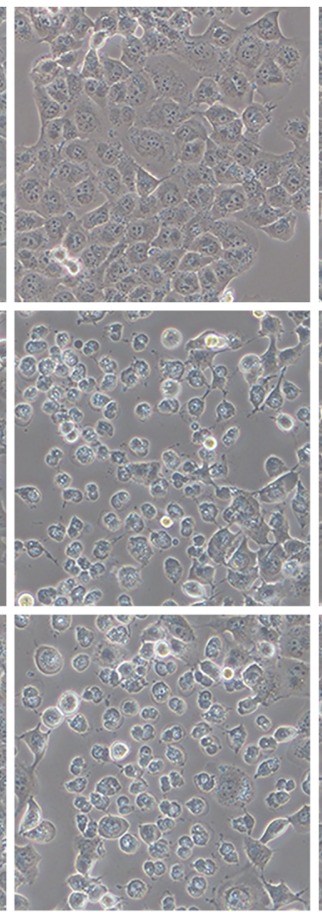

+ Era + Ferr-1
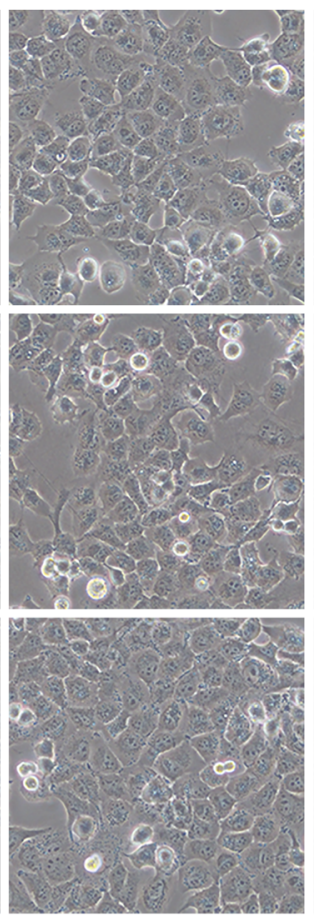
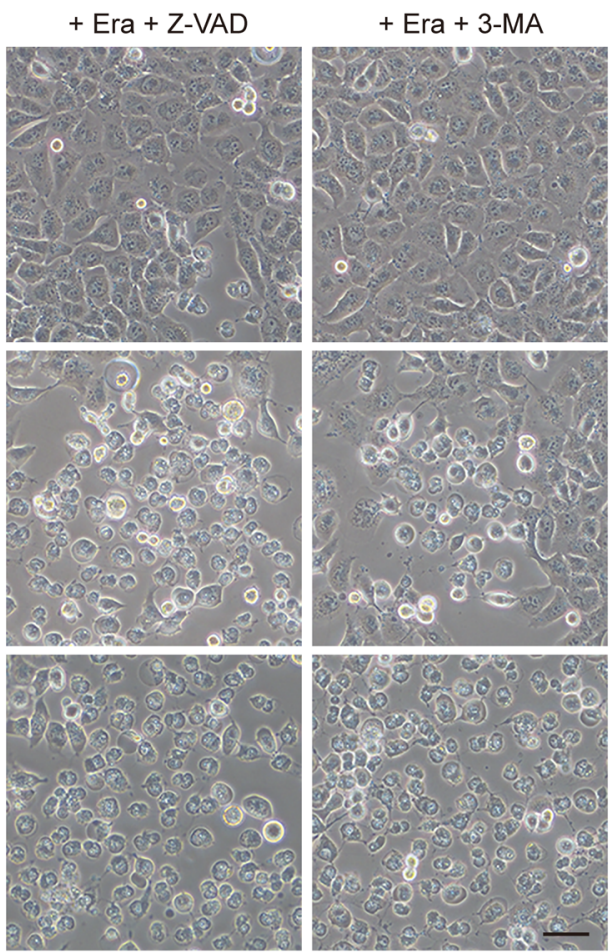

$\mathbf{F}$
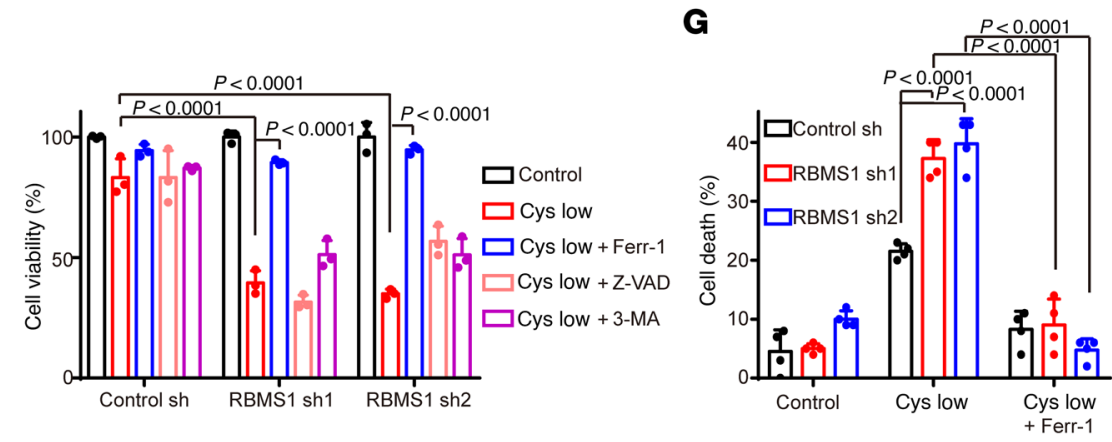
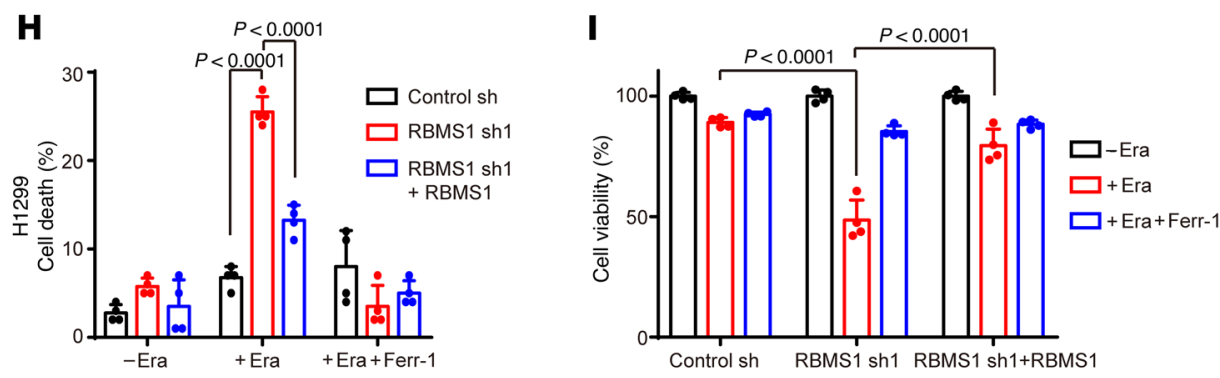
Figure 2. RBMS1 ablation promotes lung cancer cell ferroptosis. (A) Cell viability was assessed after treatment with different concentrations of erastin in RBMS1-depleted H1299 cells. (B) Cell death was measured after treatment with erastin (Era) or Ferr-1 and erastin in RBMS1-depleted H1299 cells. (C) Representative phase-contrast images of RBMS1-depleted H1299 cells treated with erastin, erastin and Ferr-1, erastin and Z-VAD-FMK (Z-VAD), or erastin and 3-methylademine (3-MA). Scale bar: $100 \mu \mathrm{m}$. (D and $\mathbf{E}$ ) Bar graphs showing viability of RBMS1-depleted $\mathrm{H} 1299$ cells treated with erastin (D) or tert-butyl hydroperoxide (TBH) (E) combined with Ferr-1, Z-VAD, or 3-MA. (F) Viability of RBMS1-depleted H1299 cells was measured after culturing in low-cystine medium combined with Ferr-1, Z-VAD, or 3-MA. (C) Bar graph showing death of BMS1-depleted H1299 cells cultured in low-cystine medium with or without Ferr-1. ( $\mathbf{H}$ and $\mathbf{I})$ Cell death $\mathbf{( H )}$ and viability (I) were measured after treatment with erastin or erastin and Ferr-1 in RBMS1-depleted $\mathrm{H} 1299$ cells with or without RBMS1 reexpression. Data represent mean \pm SEM, with $n=3$ (A and F) or 4 (B, D, E, and G-I) independent repeats. $P$ values were determined using 1-way repeated measures ANOVA (A) or 1-way ANOVA with Tukey's multiple comparison test (B, D, and $\mathbf{E}-\mathbf{I})$.

section micro-computed tomography (micro-CT), both female and male $\mathrm{Kras}^{\mathrm{G} 12 \mathrm{D} / \mathrm{WT}} / \mathrm{Rbms}^{1 / f / f} \mathrm{CKO}$ mice had markedly fewer detectable nodules than $\mathrm{Kras}^{\mathrm{G12D} / W T} / \mathrm{Rbms}^{\mathrm{WT}}$ mice in the lung (Figure 3D and Supplemental Figure 4, E and F). Further tumor analysis revealed that $\mathrm{Kras}^{\mathrm{GID} / \mathrm{WT}} / \mathrm{Rbms}^{\mathrm{fH} / \mathrm{fl}} \mathrm{CKO}$ mice showed a noticeably reduced tumor area and tumor burden as compared with $\mathrm{Kras}^{\mathrm{G12D} / W T} /$ $R b m s 1^{W T}$ mice (Figure 3E and Supplemental Figure 4G). As expected, compared with tumor tissues from $K r a s^{G 12 D / W T} / R b m s 1^{W T}$ mice, tumor sections from $\mathrm{Kras}^{\mathrm{GlD} / \mathrm{WT}} / \mathrm{Rbms}^{\mathrm{fl/l} / \mathrm{C}}$ CKO mice exhibited dramatically decreased levels of RBMS1 but increased levels of 4-hydroxy-2-noneal (4HNE, a biomarker of lipid peroxidation), as judged by IHC (Figure 3F). Therefore, consistent with our observations from clinical samples, our findings in mice support the notion that loss of RBMS1 significantly inhibits lung cancer progression, and this inhibition is associated with increased ferroptosis marker (4HNE) staining.

Loss of RBMS1 inhibits lung cancer progression in cultured cells. We further examined the RBMS1 level in a panel of lung cancer cell lines, and found that RBMS1 is markedly increased in multiple lung cancer cell lines as compared with normal bronchial cell lines (Figure 4A). Depletion of RBMS1 significantly inhibited the growth of H1299 and A549 lung cancer cells (Figure 4B and Supplemental Figure 5A). In addition, RBMS1 knockdown significantly inhibited anchorage-dependent growth in both H1299 and A549 lung cancer cells, as judged by a colony formation assay (Figure 4C and Supplemental Figure 5B). This RBMS1 knockdown-induced growth inhibition was associated with significantly suppressed proliferation in H1299 and A549 lung cancer cells, as judged by EdU staining (Figure 4D and Supplemental Figure 5C). Similar growth inhibition was also observed in RBMS1deleted H1299 and A549 lung cancer cells (Supplemental Figure 5, D-G). Again, RBMS1 deletion-induced growth suppression could be partially rescued by ferroptosis inhibitors Ferr-1 and liproxstatin-1 but not other cell death inhibitors, indicating that the depletion of RBMS1 inhibits cell growth in a ferroptosis-dependent manner (Supplemental Figure 5H). To further test whether RBMS1 reduction inhibits lung cancer cell growth in vivo, we subcutaneously injected H1299 lung cancer cells carrying a doxycycline-inducible shRNA against RBMS1 into flanks of nude mice, and then fed them water without or with doxycycline to deplete RBMS1 (Figure 4E). The growth of tumors was measured every 3 days, and xenograft tumors were removed for final analysis. Consistent with our observation in vitro, RBMS1-depleted H1299 cells grew much more slowly than controls and developed smaller tumors compared with control cells (Figure 4, F-H). Therefore, loss of RBMS1 inhibits lung cancer progression both in cultured cells and in xenograft tumors.

Depletion of RBMS1 inhibits lung cancer progression partially through a reduction in SLC7A11 and subsequent activation of ferroptosis. To better understand the molecular mechanisms underlying RBMS1-regulated ferroptosis in lung cancer, we performed quantitative proteomics assays using lung cancer cells with doxycycline-induced depletion or overexpression of RBMS1. Intriguingly, the protein level of SLC7A11, a key suppressor of ferroptosis, was significantly decreased upon RBMS1 depletion (Figure 5A, Supplemental Figure 6A, and Supplemental Table 1). We confirmed that the expression of SLC7A11 was markedly reduced in the RBMS1-depleted H1299 and A549 lung cancer cells by either stable or transient knockdown; however, the levels of other ferroptosis regulators, including ACSL4, AIFM2, GPX4, and DHODH (35), were not affected (Figure 5B and Supplemental Figure 6, B and C). Conversely, stable overexpression of RBMS1 increased the expression level of SLC7A11 but not other ferroptosis regulators, in both H1299 and A549 cells (Figure 5B and Supplemental Figure 6C). In addition, reexpression of RBMS1 in lung cancer cells with stable RBMS1 depletion rescued the RBMS1 reduction-induced decrease in SLC7A11 (Supplemental Figure 6D). Since SLC7A11 regulates the uptake of extracellular cystine, a major precursor for GSH biosynthesis, we subsequently examined the cystine uptake and found that knockdown of RBMS1 significantly decreased the uptake of extracellular cystine (Figure $5 \mathrm{C}$ ) and significantly decreased the levels of GSH (Figure 5D). Similar results were also obtained in H1299 lung cancer cells with doxycycline-induced depletion of RBMS1 (Supplemental Figure 6E). In addition, depletion of RBMS1 notably increased lipid peroxidation (Figure 5E). Importantly, restoration of SLC7A11 in RBMS1-knockdown or RBMS1-deleted H1299 lung cancer cells almost fully suppressed erastin-triggered cell death (Figure 5, F-H, and Supplemental Figure 6, F-I).

Notably, reexpression of SLC7A11 in RBMS1-depleted H1299 lung cancer cells inhibited lipid peroxidation triggered by low-cystine medium (Figure 6A and Supplemental Figure 7A) and reversed the low-cystine-medium-induced cell death (Figure 6, B and C). Moreover, addition of $\mathrm{N}$-acetylcysteine (NAC) to RBMS1-depleted cells with cystine deficiency could rescue the RBMS1 depletion-induced cancer cell growth inhibition, suggesting that such an effect is dependent on cystine uptake via SLC7A11 (Supplemental Figure 7B). Taken together, our data indicate that RBMS1 depletion promotes ferroptosis mainly through repressing SLC7A11 expression, and further suggest that decreased RBMS1 might inhibit lung cancer progression at least partly through suppression of SLC7A11 and promotion of ferroptosis.

To test this hypothesis, we stably transfected RBMS1-depleted H1299 and A549 lung cancer cells with a construct expressing SLC7A11 or a control vector (Supplemental Figure 7C). Restoration of SLC7A11 in stably RBMS1-depleted lung cancer cells partially restored RBMS1 knockdown-induced suppression of cell growth 
A
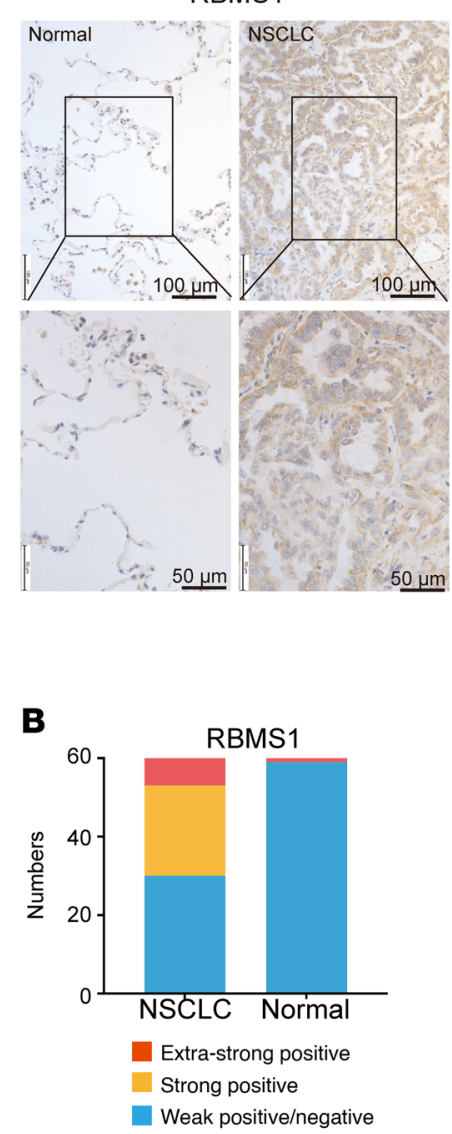

C

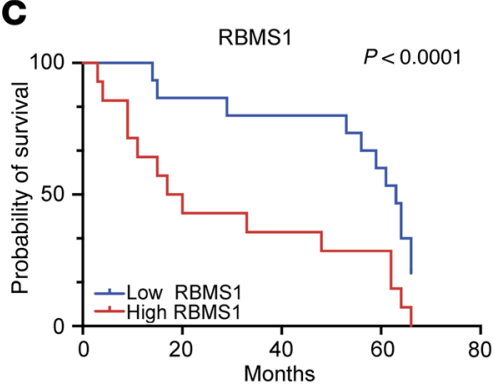

E

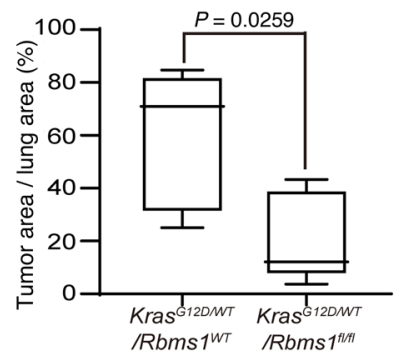

D

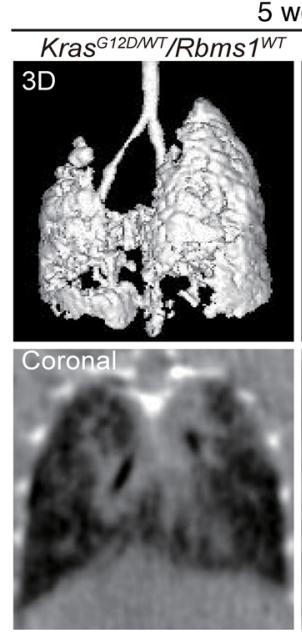

5 weeks
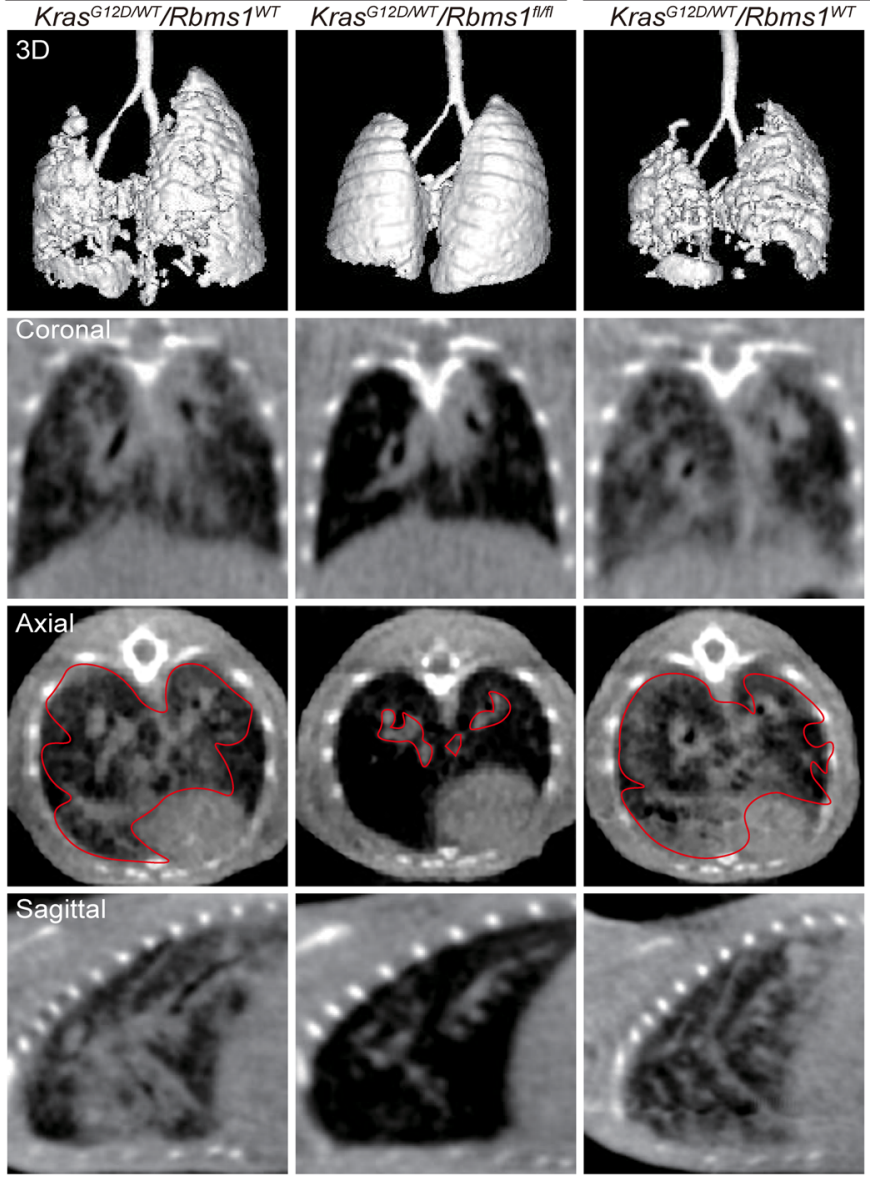

Female

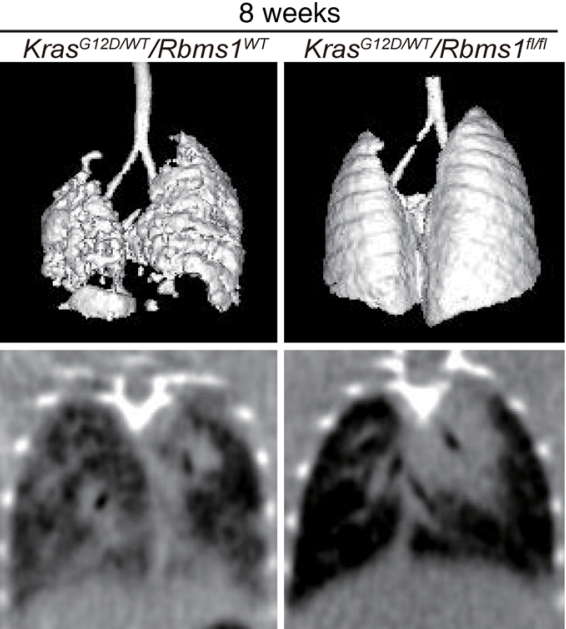

F
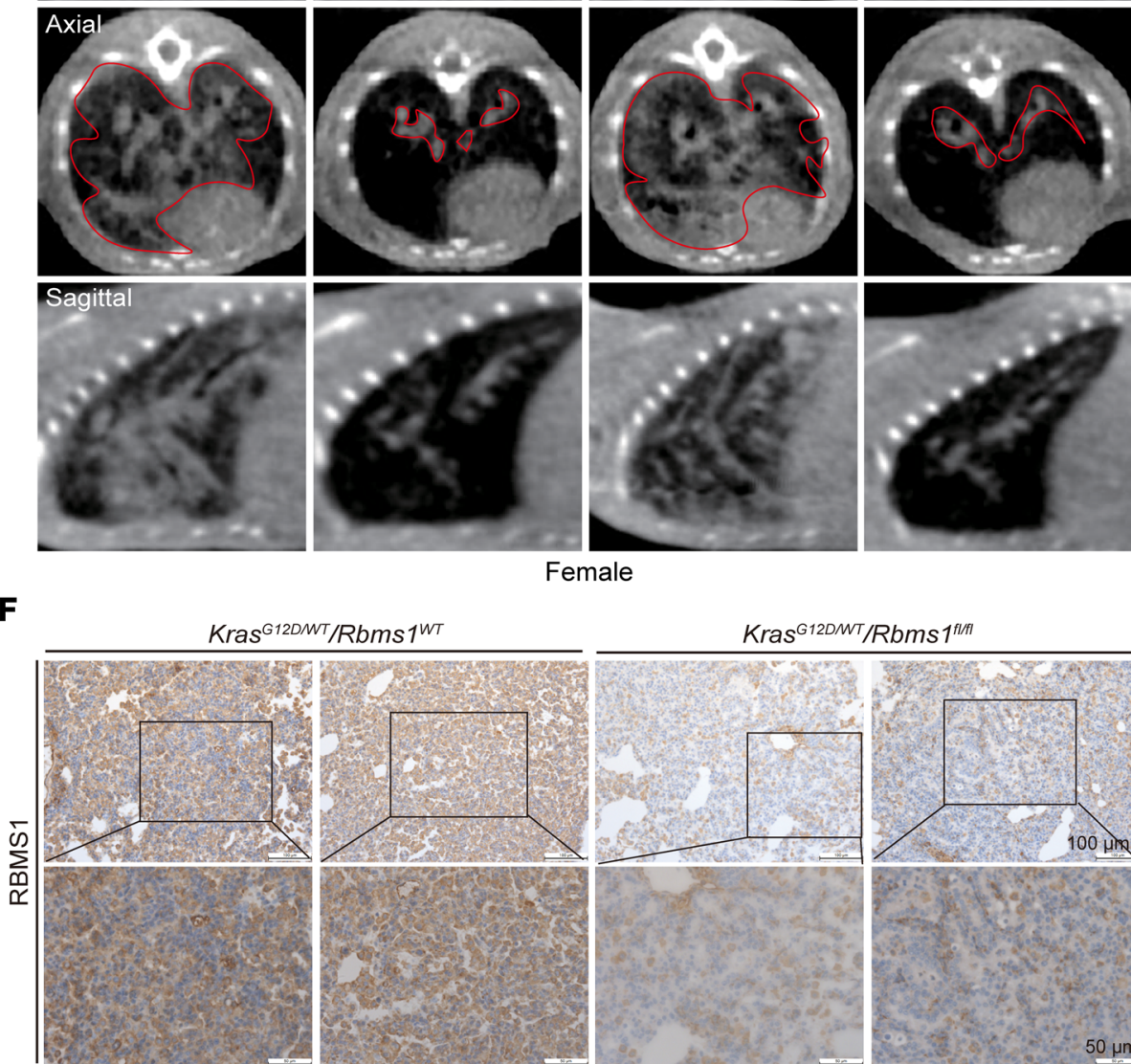

$K_{r a s}^{G 12 D N T} / R b m s 1^{\text {Alfit }}$
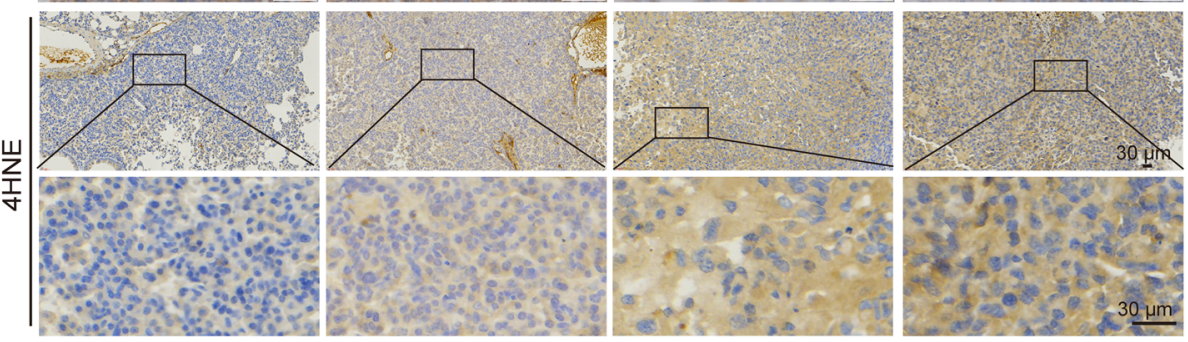
Figure 3. RBMS1 is associated with lung cancer progression and prognosis in humans and mice. (A) Representative images from immunohistochemical staining of RBMS1 in lung cancer $(n=60)$ and matched adjacent normal tissues $(n=60)$. Scale bars: $100 \mu \mathrm{m}$ (top) and $50 \mu \mathrm{m}$ (bottom). (B) The quantification of RBMS1 protein level in lung cancer and adjacent normal lung tissues. The RBMS1 levels were classified into 3 grades (weak positive/ negative, strong positive, extra-strong positive) based on quantification of immunohistochemical staining and plotted. (C) Kaplan-Meier curve showing overall survival of lung cancer patients with high or low RBMS1 expression. (D) Micro-CT images in the indicated planes from female mice with or without

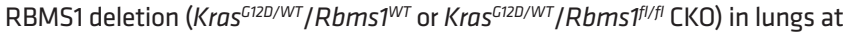
5 or 8 weeks after infection with $9 \times 10^{10} \mathrm{vg}$ AAV-GFP-Cre. Three-dimensional rendering of micro-CT data shows lungs in gray. The lung tumor areas of

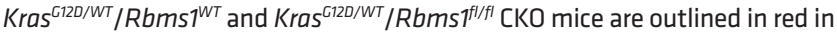

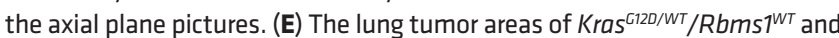

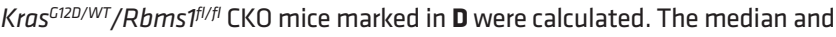
upper and lower quartiles of tumor areas were plotted as box-and-whisker plot ( $n=5, P$ values from unpaired $t$ test). (F) Tumors were removed from $\mathrm{Kras}^{\mathrm{C120/WT}} / \mathrm{Rbms} \mathrm{T}^{\mathrm{WT}}$ and $\mathrm{Kras}^{\mathrm{G}}{ }^{12 D / W T} / \mathrm{Rbms} 7^{\mathrm{fl} / \mathrm{fl}} \mathrm{CKO}$ mice and subjected to immunohistochemical staining with anti-RBMS1 and anti-4HNE antibodies. Representative images $(n=5)$ are shown. Scale bars: $100 \mu \mathrm{m}$ (top row), $50 \mu \mathrm{m}$ (second row), and $30 \mu \mathrm{m}$ (rows 3 and 4 ).

(Figure 6D and Supplemental Figure 7D). In addition, the proliferative ability of RBMS1-depleted lung cancer cells was significantly promoted by SLC7A11 reexpression, as judged by colony formation assay and EdU staining (Figure 6, E and F, and Supplemental Figure 7, E and F). Consistently, RBMS1 depletion-induced inhibition of xenograft tumor development was also significantly rescued with restoration of SLC7A11 or treatment with the ferroptosis inhibitor liproxstatin-1, suggesting that such tumor suppression is also dependent on ferroptosis in the xenograft mouse model (Figure 6, G-I, and Supplemental Figure 7, G and H). Further examination of lipid peroxidation by IHC staining for $4 \mathrm{HNE}$ revealed that RBMS1-depleted tumors had increased 4HNE staining compared with control tumors, but this increase was markedly reduced upon reexpression of SLC7A11 (Figure 6J). Together, our results indicate that loss of RBMS1 suppresses lung cancer progression partially through repressing SLC7A11 and promoting ferroptosis both in vitro and in vivo.

RBMS1 regulates the translation of SLC7A11. We next aimed to understand how depletion of RBMS1 suppresses the expression of SLC7A11. Previously, RBMS1 has been shown to function as a posttranscriptional regulator of RNA stability to modulate gene expression (33). We thus sought to investigate whether RBMS1 affects the mRNA expression of SLC7A11. Surprisingly, the mRNA level of SLC7A11 was not affected by RBMS1, as judged by quantitative real-time PCR (qRT-PCR) in both the stably RBMS1-depleted lung cancer cells and in those with RBMS1 transiently knocked down with siRNA (Figure 7A and Supplemental Figure 8A). This observation suggests that RBMS1 might regulate the expression of SLC7A11 at the post-RNA level, including translation.

To directly examine whether RBMS1 regulates the translation of SLC7A11, we generated 4 translation reporters using the promoter and different UTR fragments from SLC7A11: SLC7A11-flucFL (promoter region, 5'-UTR, and 3'-UTR); SLC7A11-fluc-T1 (promoter region and $5^{\prime}$-UTR); SLC7A11-fluc-T2 (promoter region, $5^{\prime}$-UTR, and nt 1-3846 of 3'-UTR); and SLC7A11-fluc-T3 (promoter region, 5'-UTR, and nt 3827-7859 of 3'-UTR) (Figure 7B). We conducted luciferase reporter assays and found that the activity of luciferase reporter SLC7A11-fluc-FL was significantly inhibited by depletion of RBMS1 in lung cancer cells; however, the mRNA level of SLC7A11-fluc-FL was not affected (Figure 7C). Moreover, only the activity of luciferase reporter SLC7A11-fluc-T3, but not that of SLC7A11-fluc-T1 and SLC7A11-fluc-T2, was significantly suppressed in RBMS1-depleted lung cancer cells (Figure 7D and Supplemental Figure 8B), suggesting that RBMS1 regulates the translation of SLC7A11 protein through the second half of its 3'-UTR (the T3 region). In support of this idea, multiple RBMS1 binding sites (e.g., AU-rich sequences) were found in the T3 region, and truncated mutations of partial binding sites (SLC7A11-fluc-T4 or SLC7A11-fluc-T5) could not totally abolish such repressive function (Supplemental Figure 8C). Additionally, reexpressing RBMS1 reversed the RBMS1 depletion-induced suppression of the luciferase activity of SLC7A11-fluc-T3 (Figure 7E and Supplemental Figure 8D). We further analyzed actively translated mRNAs by ribosome profiling (Ribo-seq) analysis and identified a number of actively translated mRNAs whose translation might be controlled by RBMS1, with SLC7A11 as one of the top targets (Figure 8). Consistent with this observation, immunofluorescence assays showed that RBMS1 was colocalized with calnexin, an ER marker, suggesting that RBMS1 might localize at the ER to regulate translation (Supplemental Figure 8E). Together, our results indicate that RBMS1 is capable of promoting the translation of SLC7A11 through the T3 region of its 3'-UTR.

RBMS1 interacts with eIF3d to bridge the 3'- and 5'-UTR of SLC7A11 to promote its translation. To better understand how RBMS1 regulates the translation of SLC7A11, we pulled down RBMS1-containing immunocomplexes from cells stably expressing RBMS1 and determined its associated proteins using mass spectrometry. Cells containing empty vector were used as controls. In total, 118 proteins were specifically identified. When analyzing cellular functions of potential RBMS1-interacting proteins using Gene Ontology (GO), we found that the interacting proteins were mostly enriched in multiple translation-related pathways, including translation regulation, translation initiation, and ribonucleoprotein biogenesis (Figure 9A). Further STRING analysis (https://string-db.org/) revealed that RBMS1-interacting proteins were functionally clustered into a few well-linked interaction networks, including the expected RNA processing subgroup (Figure 9B). However, remarkably, proteins involved in the regulation of translation formed the other major subgroup (Figure 9B). Therefore, our results further demonstrate that RBMS1 might regulate the expression of SLC7A11 through control of translation.

Strikingly, multiple key translation regulators, including cap-binding protein eukaryotic initiation factor 3d (eIF3d), eIF3i, and eIF3m, were identified in the immunoprecipitation-coupled (IP-coupled) mass spectrometry assay using exogenously expressed RBMS1 as a bait. These factors are critical components of the eIF3 complex that could drive a cap-dependent pathway of translation initiation (36). We confirmed that exogenously expressed eIF3d, eIF3i, and eIF3m could independently pull down endogenous RBMS1 in a co-IP assay (Figure 9C). Inversely, exogenously expressed RBMS1 pulled down endogenous eIF3d, eIF3i, and eIF3m in both H1299 and A549 cells as well (Figure 9D and Supplemental Figure 9A). The expression levels of eIF3d, eIF3i, 
A

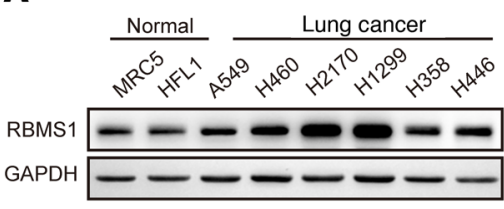

B

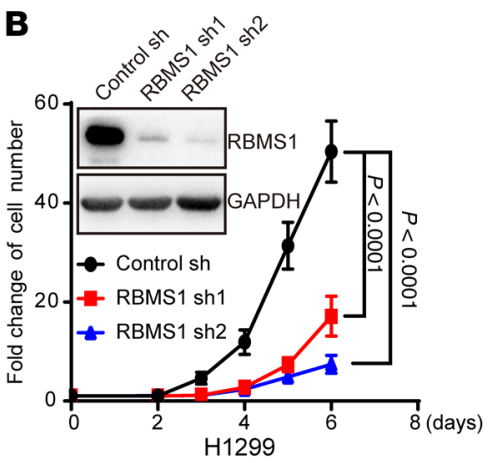

D
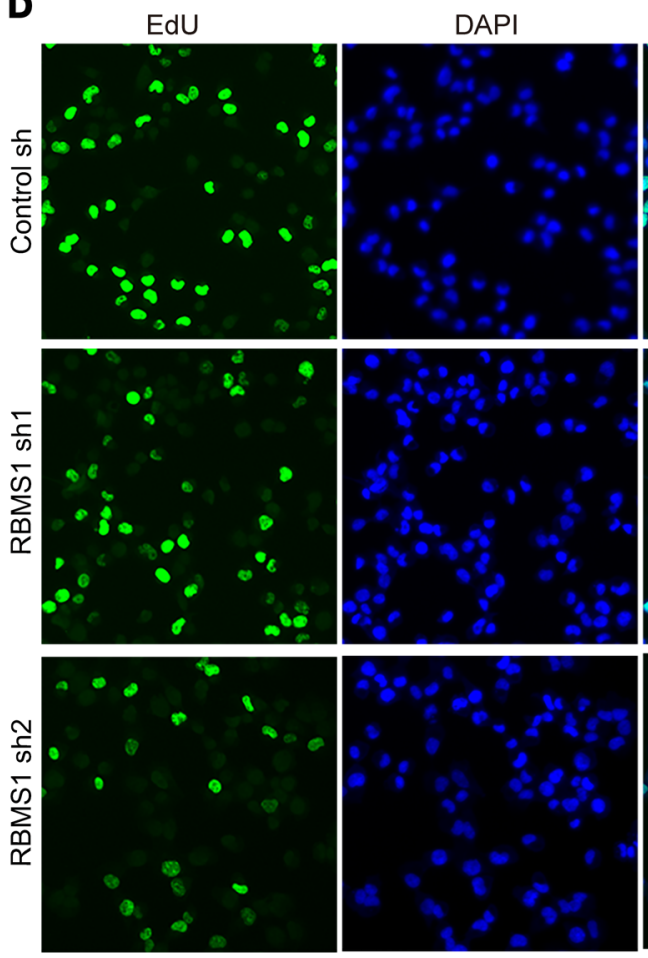
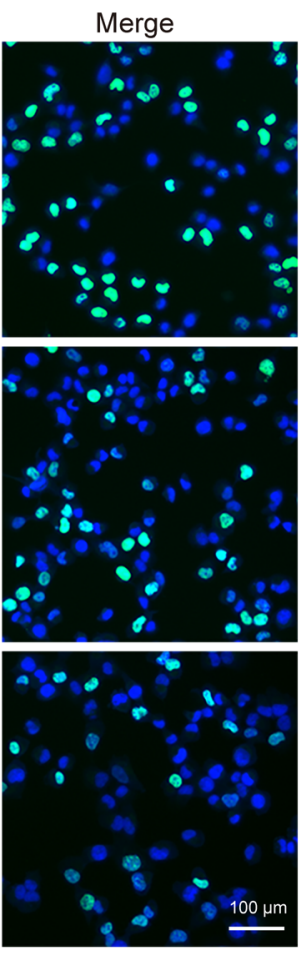
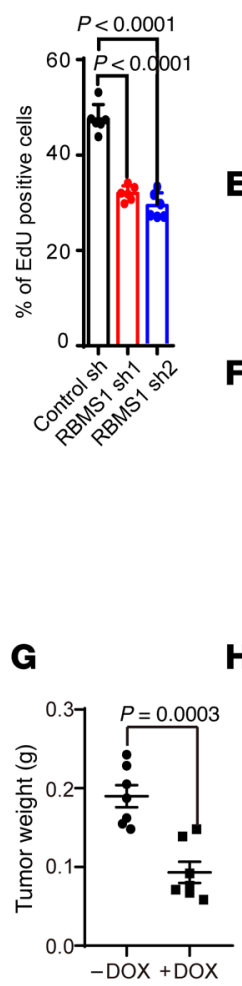
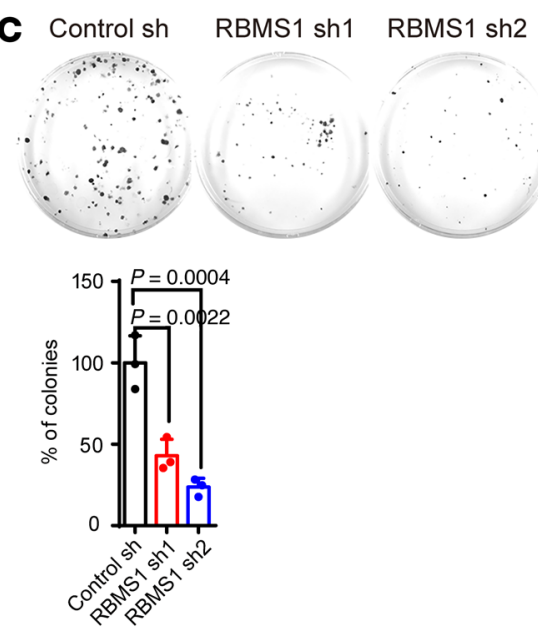

E

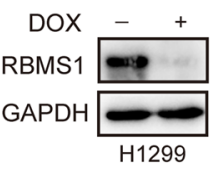

$\mathbf{F}$

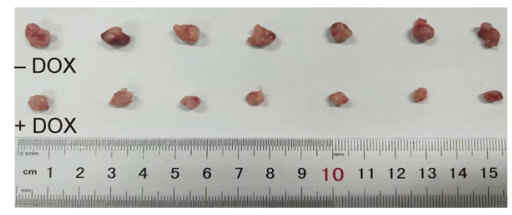

H

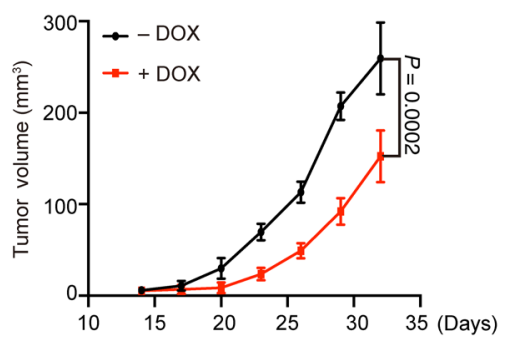

Figure 4. Loss of RBMS1 inhibits lung cancer cell proliferation and progression in vitro and in vivo. (A) Levels of RBMS1 in the indicated lung cancer cell lines and normal bronchial cell lines were measured by Western blot assay. (B) H1299 cells with stable depletion of RBMS1 or control were grown for 6 days, with cell numbers counted every 2 days. The changes in cell numbers were compared to day 0 , and the mean \pm SEM from 3 experiments was plotted. (C) Colony formation assays using H1299 cells with stable depletion of RBMS1. Images of the whole plate are shown. Three experiments were carried out, with mean \pm SEM of relative colony numbers plotted. (D) The proliferative abilities of stably RBMS1-depleted H1299 cells were measured with an EdU staining assay. Six experiments were conducted, with mean \pm SEM of percentage of EdU-positive cells plotted. Scale bar: $100 \mu \mathrm{m}$. (E) The protein levels of RBMS1 in H1299 cells with doxycycline-inducible depletion of RBMS1 were examined in the absence or presence of doxycycline by Western blot assay. (F-H) Xenograft tumors were generated using nude mice subcutaneously injected with $\mathrm{H} 1299$ cells with doxycycline-inducible depletion of RBMS1. Mice were fed water with or without doxycycline. (F) Pictures of the tumors removed after 32 days. (G) Tumors were weighed and plotted. (H) The average sizes of xenograft tumors were measured every 3 days and plotted $(n=7$, error bars indicate mean \pm SEM). $P$ values were determined by 1 -way repeated measures ANOVA (B and $\mathbf{H}$ ), 1-way ANOVA with Dunnett's multiple comparison test (C and $\mathbf{D})$, or unpaired $t$ test (G).

and eIF3m were not affected by RBMS1 (Supplemental Figure 9B). Interestingly, the interaction between RBMS1 and eIF3m was dependent on RNA, as RNase treatment abolished their interaction in a co-IP assay (Figure 9E). Furthermore, an in vitro pull-down assay using purified proteins showed that GST-RBMS1 pulled down His-eIF3d, but not His-eIF3i or His-eIF3m, indicating that RBMS1 is able to directly interact with eIF3d (Figure 9F and Supplemental Figure 9C). Importantly, proximity ligation assay (PLA), a powerful tool that allows in situ detection of endog- enous protein interactions with high specificity and sensitivity, confirmed that RBMS1 indeed interacted with endogenous eIF3d in the cytoplasm (Figure 9G and Supplemental Figure 9D). Taken together, our data suggest that RBMS1 might regulate the translation of SLC7A11 through directly interacting with eIF3d.

To test this possibility, we overexpressed eIF3d together with the luciferase reporters of SLC7A11. As expected, overexpression of eIF3d significantly promoted the activity of luciferase reporter SLC7A11-fluc-T3, but not SLC7A11-fluc-T2, in lung cancer cells 
A

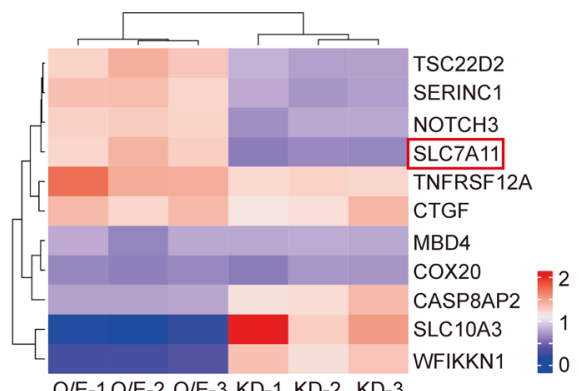

O/E-1 O/E-2 O/E-3 KD-1 KD-2 KD-3
B

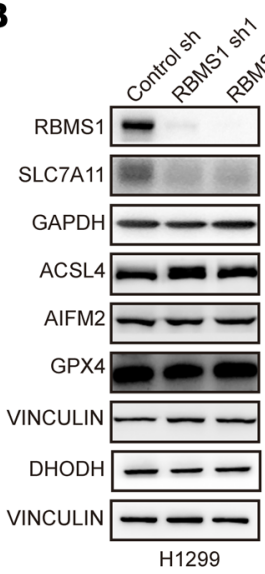

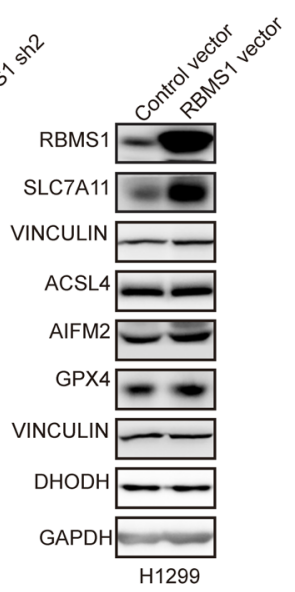

G

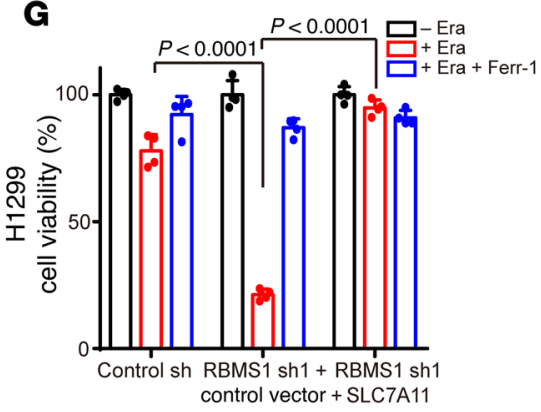

C

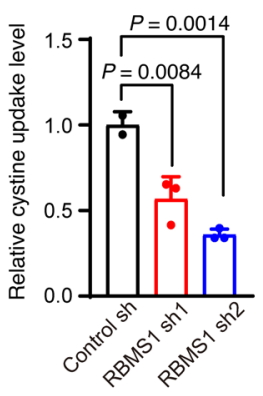

D

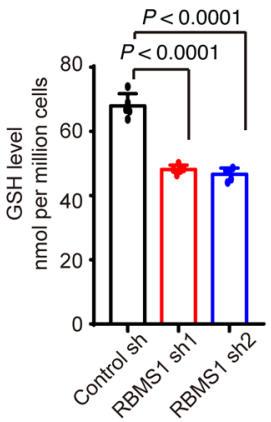

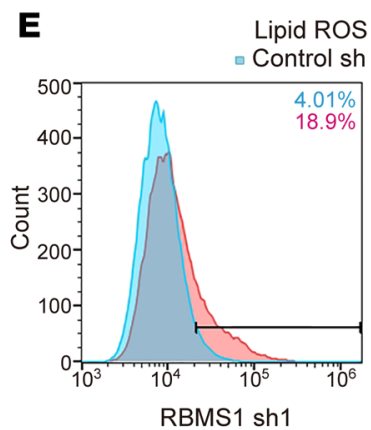
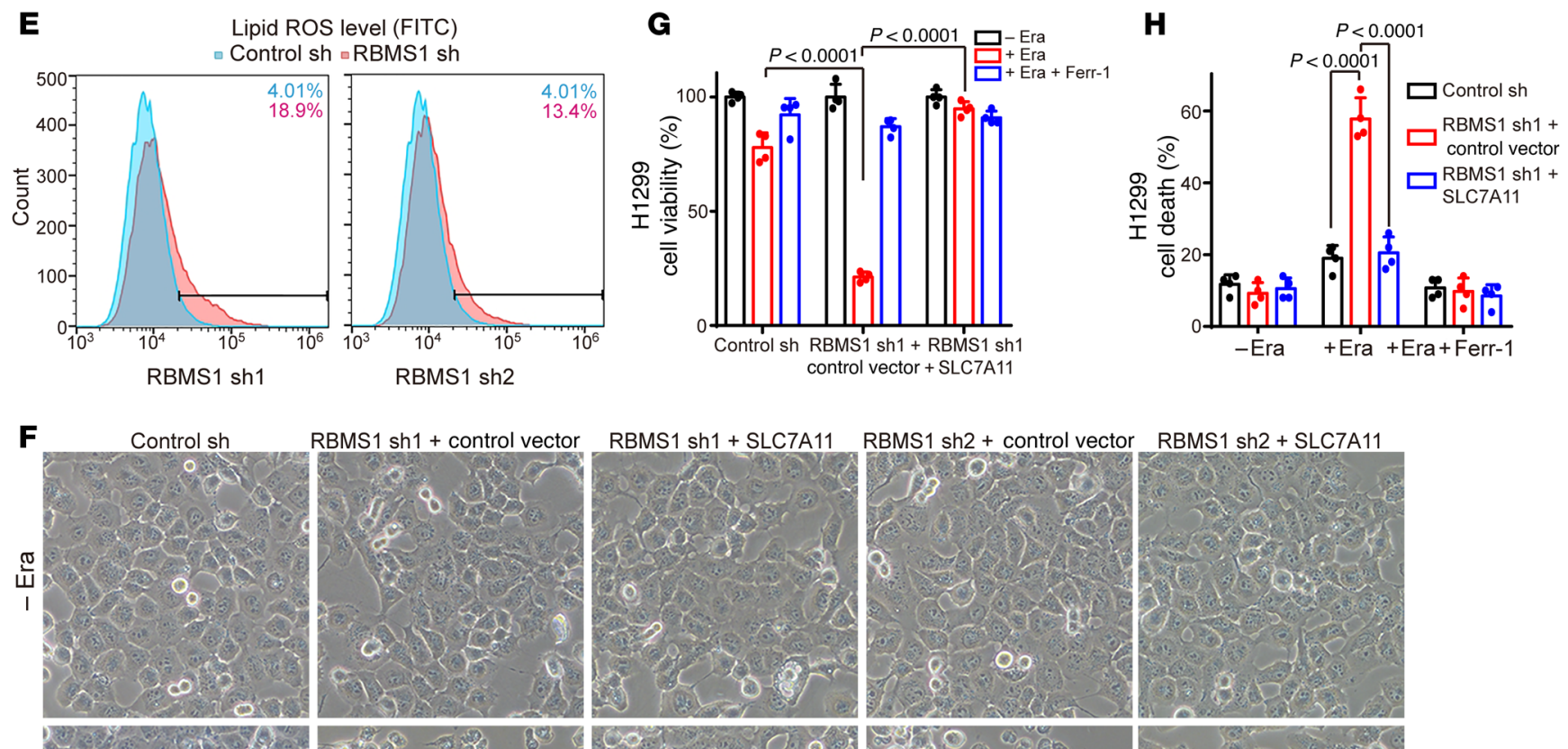

RBMS1 sh2 + SLC7A11
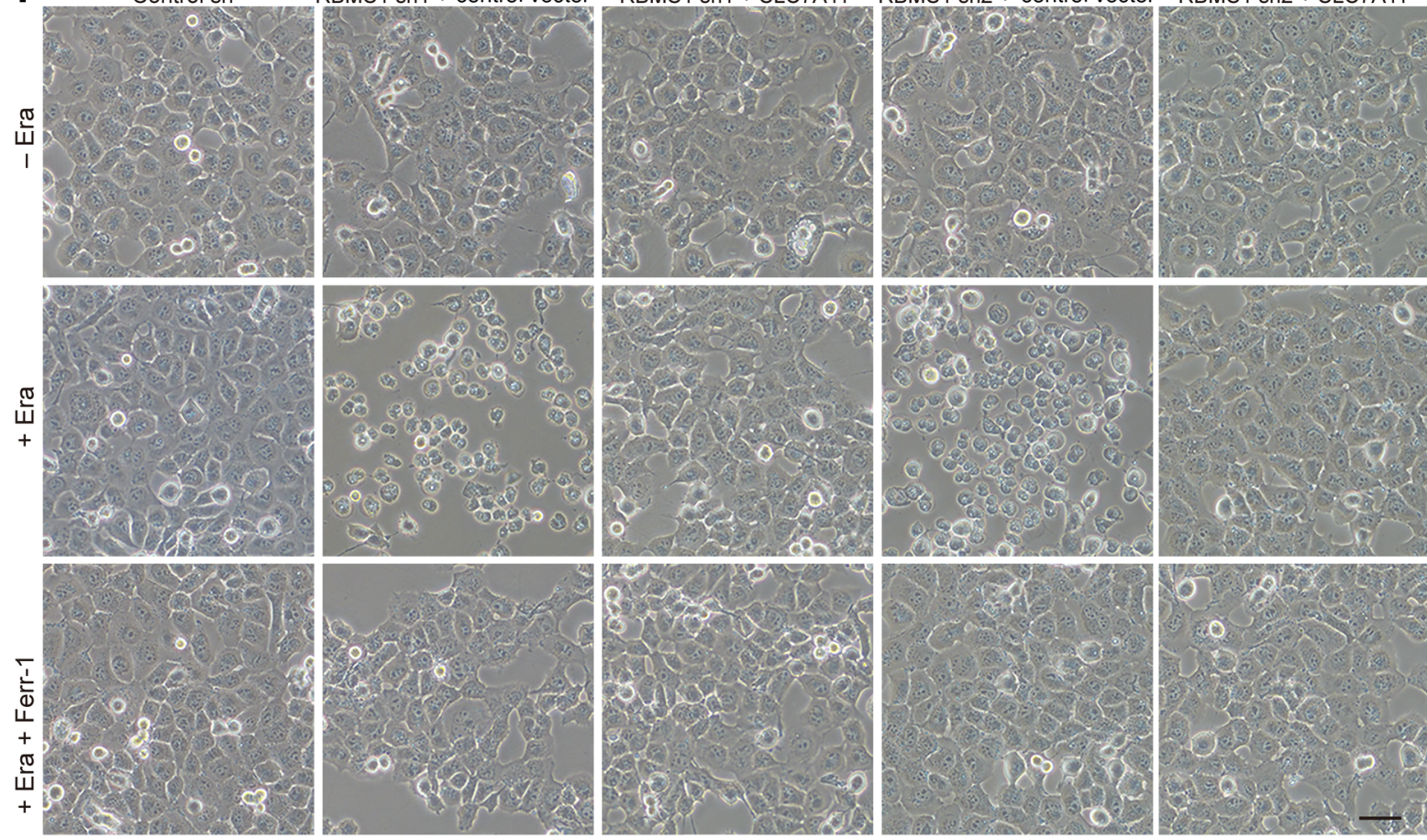

$\mathrm{H} 1299$

Figure 5. Depletion of RBMS1 stimulates ferroptosis through decreased SLC7A11. (A) Heatmap depicting levels of the most differentially expressed RBPs from quantitative proteomics using A549 cells with depleted or overexpressed RBMS1. (B) Levels of ferroptosis-related genes were examined in RBMS1-depleted or -overexpressing $\mathrm{H} 1299$ cells. (C) The relative level of cystine uptake was measured in RBMS1-depleted H1299 cells. (D) Bar graph demonstrating intracellular CSH levels in RBMS1-depleted H1299 cells. (E) Lipid peroxidation was measured by flow cytometry after C11-BODIPY staining in RBMS1-depleted H1299 cells. (F) Representative phase-contrast images of RBMS1-depleted H1299 cells, with or without SLC7A11 reexpression, treated with erastin or erastin and Ferr-1. Scale bar: $100 \mu \mathrm{m}$. (G and $\mathbf{H})$ Bar graphs showing viability $\mathbf{( G )}$ and death $(\mathbf{H})$ of H1299 cells with RBMS1 knockdown, with or without SLC7A11 reexpression and treated with erastin or erastin and Ferr-1. Data represent mean $\pm S E M, n=3$ (C and $\mathbf{D})$ or $4(\mathbf{G}$ and $\mathbf{H})$ independent repeats. $P$ values were determined by 1-way ANOVA with Dunnett's multiple comparison test (C and $\mathbf{D}$ ) or 1-way ANOVA with Tukey's multiple comparison test (G and $\mathbf{H})$. 
A

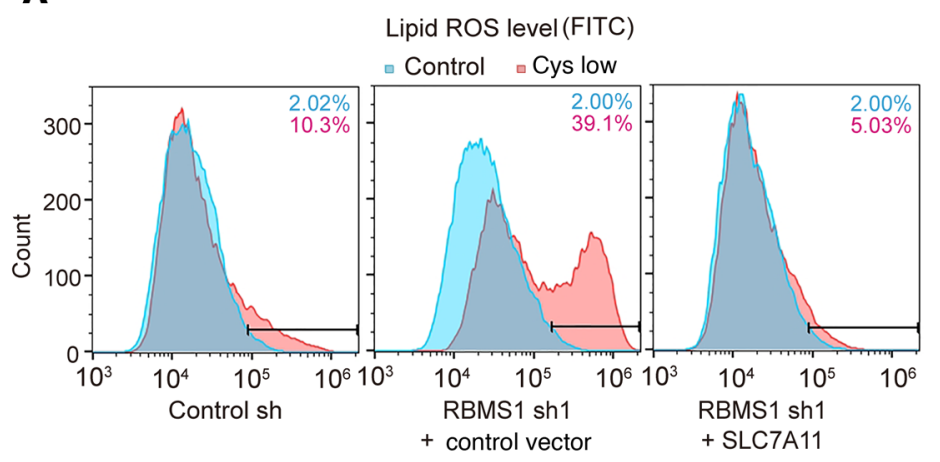

D

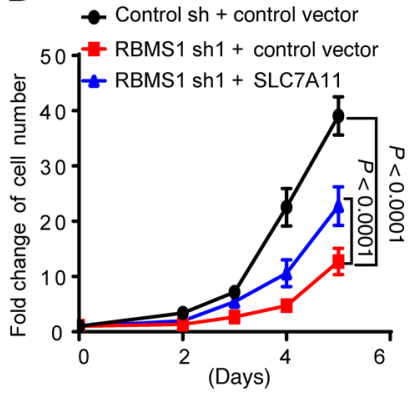

E

Control sh + RBMS1sh1 + RBMS1 sh1 + control vector control vector SLC7A11

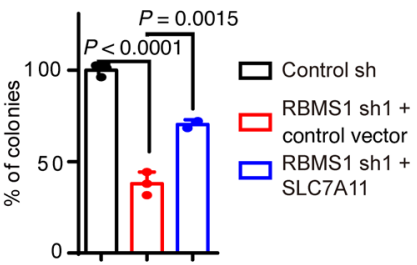

G

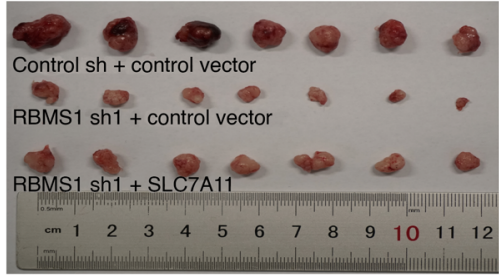

\section{$\mathbf{F}$}
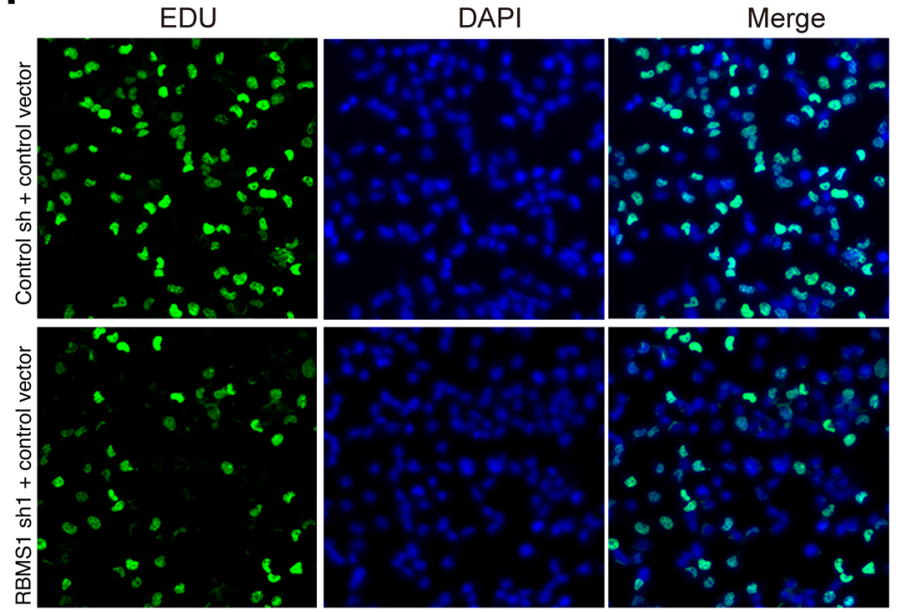

$\square$ Control $\square$ Cys low $\square+\begin{gathered}\text { Cys low } \\ + \text { Ferr-1 }\end{gathered}$
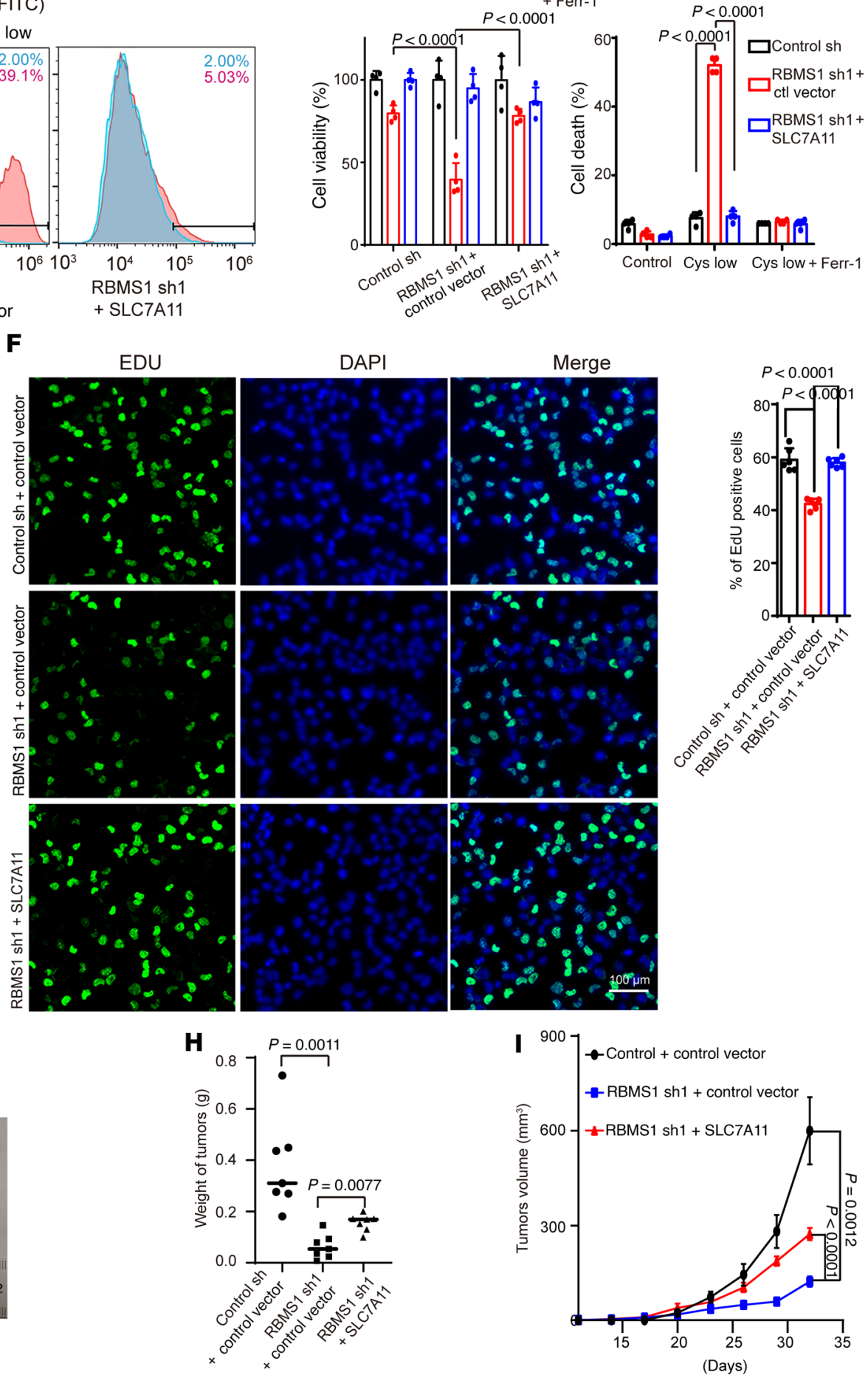

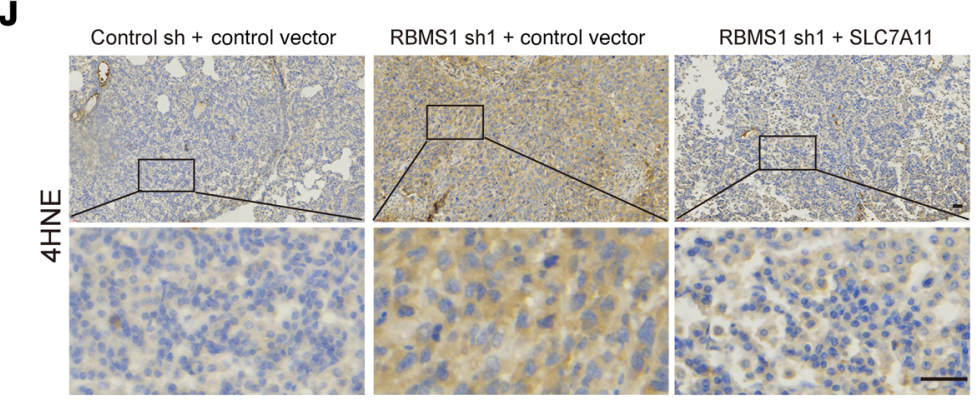


Figure 6. Depletion of RBMS1 inhibits lung cancer progression partially through decreasing SLC7A11 and promoting ferroptosis. (A) Lipid peroxidation was measured in RBMS1-depleted H1299 cells, with or without SLC7A11 reexpression, cultured in low-cystine medium. (B and C) Viability (B) and death (C) of RBMS1-depleted H1299 cells, with or without SLC7A11 reexpression, were measured after culturing in low-cystine medium combined with Ferr-1. (D-F) Growth (D), colony formation (E), and proliferative abilities (F) of RBMS1-depleted H1299 cells, with or without SLC7A11 reexpression, were measured. Scale bar: $100 \mu \mathrm{m}$. (G and $\mathbf{H})$ RBMS1-knockdown H1299 cells, with or without SLC7A11 reexpression, were subcutaneously injected into the flanks of nude mice. (C) Pictures of the removed tumors. (H) Tumors were weighed and plotted. (I) The average sizes of xenograft tumors were measured $(n=7)$. (J) Immunohistochemical staining of $4 \mathrm{HNE}$ in tumor from RBMS1-depleted H1299 cells, with or without SLC7A11 reexpression. Scale bars: $30 \mu \mathrm{m}$. Data represent mean $\pm \mathrm{SEM}, n=3$ (D), 4 (B and $\mathbf{C}$ ), or 6 (F) independent repeats. $P$ values were determined by 1-way ANOVA with Tukey's multiple comparison test (B, C, E, F, and $\mathbf{H}$ ) or 1-way repeated measures ANOVA ( $\mathbf{D}$ and $\mathbf{I}$ ).

(Figure 10A and Supplemental Figure 9E). Interestingly, eIF3d-induced activation of SLC7A11-fluc-T3 reporter was abolished in RBMS1-depleted cells (Figure 10B and Supplemental Figure 9F), indicating that the translational activation of SLC7A11 by eIF3d is dependent on RBMS1. Conversely, depletion of eIF3d noticeably reduced the protein level of SLC7A11, and suppressed the RBMS1-induced increase in SLC7A11 (Figure 10, C and D). Collectively, our results demonstrate that eIF3d plays a vital role in RBMS1-regulated SLC7A11 translation.

We next sought to study how RBMS1 coordinates with eIF3d to regulate the translation of SLC7A11. Generally, translational regulators bind to the $3^{\prime}$ - or 5'-UTR of target genes to modulate translation. We therefore performed an RNA-IP assay with A549 and 293T cells stably overexpressing RBMS1 or eIF3d. RBMS1 and eIF3d bound to both the 3'- and 5'-UTR of SLC7A11 in the RNA-IP assay (Figure 10, E and F, and Supplemental Figure 9G). However, depletion of RBMS1 largely abolished the binding of eIF3d to the 3 '-UTR, but not the 5'-UTR of SLC7A11, suggesting that eIF3d binds to the 3'-UTR of SLC7A11 through interacting with RBMS1 (Figure 10G and Supplemental Figure 9H). Additionally, knockdown of eIF3d almost completely disrupted the binding of RBMS1 to the 5'-UTR, but not the 3 '-UTR of SLC7A11, indicating that RBMS1 binds to the $5^{\prime}$-UTR of SLC7A11 via interacting with eIF3d (Figure $10 \mathrm{H}$ and Supplemental Figure 9I). Thus, these data suggest that eIF3d is associated with the $3^{\prime}$-UTR of SLC7A11 through RBMS1, whereas RBMS1 binds to the 5'-UTR of SLC7A11 through eIF3d. Taken together, our results support a model in which RBMS1 binds to the eIF3d complex to bridge the $3^{\prime}$ - and 5'-UTR of SLC7A11, which in turn promotes the translation of SLC7A11 (Figure 10I).

RBMS1 positively correlates with elevated SLC7A11 in clinical samples and confers radiotherapy resistance to lung cancer cells. Since RBMS1 depletion-induced translational repression of SLC7A11 promotes ferroptosis and inhibits lung cancer progression, we examined the expression of RBMS1 and SLC7A11 in surgically collected, paired, non-small cell lung cancer (NSCLC) samples and adjacent normal tissues from 7 patients. Remarkably, all NSCLC specimens had markedly increased RBMS1 and SLC7A11 protein levels as compared with matched adjacent normal tissues (Figure 11A), and the level of SLC7A11 was positively correlated with that of RBMS1 in clinical samples $\left(R^{2}=0.893, P<0.0001\right.$; Figure 11B). Such observa- tion was validated by IHC using the same tissue microarrays used in Figure 3A (Figure 11C and Supplemental Figure 10A). Importantly, more than $50 \%$ of lung cancer samples showed strong or extra-strong staining for SLC7A11; however, only less than $5 \%$ of adjacent lung tissues displayed strong staining for SLC7A11 (Figure 11D). In addition, lung cancer patients with a higher expression level of SLC7A11 likely had a higher clinical stage tumor (Supplemental Figure 10B), suggesting that the expression level of SLC7A11 is positively correlated with the clinical stage of lung cancer patients. Moreover, lung cancer patients with a relative high level of SLC7A11 had shorter overall survival than those with a low level of SLC7A11 (Figure 11E). Therefore, our clinical observations reveal that RBMS1 is positively correlated with SLC7A11 in lung cancer patient samples, further demonstrating that the clinical value of RBMS1 in lung cancer is through regulating ferroptosis.

In addition, tumor sections from $\mathrm{Kras}^{\mathrm{Gl2D} / W T} / \mathrm{Rbms}^{1 / \mathrm{fl} l} \mathrm{CKO}$ mice showed noticeably reduced levels of SLC7A11 than those in tumor tissues from $\mathrm{Kras}^{\mathrm{G} 12 \mathrm{D} / W T} / \mathrm{Rbms}^{W T}$ mice, as judged by IHC (Figure 11F), further confirming that RBMS1 deficiency-inhibited lung cancer development is associated with reduced expression of SLC7A11 in mice. We also obtained mouse embryonic fibroblasts (MEFs) from $R b m s 1^{f / f l}$ mice and found that RBMS1-deleted MEFs had a notable decrease in SLC7A11, as judged by Western blot assay (Figure 11G). Functionally, deletion of RBMS1 significantly promoted erastin-induced cell death, which could be prevented by Ferr-1, in MEF cells (Figure 11H). Therefore, our data support the notion that RBMS1 facilitates lung cancer progression partially through SLC7A11-induced evasion of ferroptosis in mice.

Radiotherapy is one of the most effective routes for lung cancer therapy. It has been previously shown that ionizing radiation (IR) induces ferroptosis, and that SLC7A11 is able to promote radioresistance mainly through suppressing ferroptosis (15). Our observation that SLC7A11 is under translational control of RBMS1 in lung cancer cells and mice raises the possibility that depletion of RBMS1 could partly sensitize IR-resistant lung cancer cells to radiotherapy through suppressing SLC7A11 expression and promoting ferroptosis. To test this possibility, we employed IR-resistant and parental A549 lung cancer cells (Supplemental Figure 10C and ref. 37) and found that the expression level of RBMS1 was increased in IR-resistant A549 cells regardless of irradiation (Figure 12A). The level of SLC7A11 was also elevated in the IR-resistant cells as compared with the parental cells (Figure 12A). Interestingly, knockdown of RBMS1 in IR-resistant A549 cells decreased the level of SLC7A11 (Figure 12B), induced more lipid peroxidation (Figure 12C), and significantly sensitized these cells to irradiation (Figure 12D). Again, Ferr-1 treatment reversed the loss of RBMS1-induced radiation sensitization in IR-resistant A549 cells (Figure 12E). Similarly, knockdown of RBMS1 had synergistic effects with irradiation on the radiosensitivity of H1299 cancer cells, which was abrogated by reexpression of SLC7A11 (Supplemental Figure 10, D and E). Collectively, our data demonstrate that RBMS1 positively correlates with elevated SLC7A11 in clinical samples and confers radiotherapy resistance to lung cancer cells. Our findings further suggest that the RBMS1/SLC7A11 axis is a potential therapeutic target for sensitization of lung cancer to radiotherapy.

Nortriptyline hydrochloride inhibits RBMS1 expression and sensitizes IR-resistant lung cancer cells to irradiation through promoting ferroptosis. To further evaluate the therapeutic potential of the RBMS1/ 

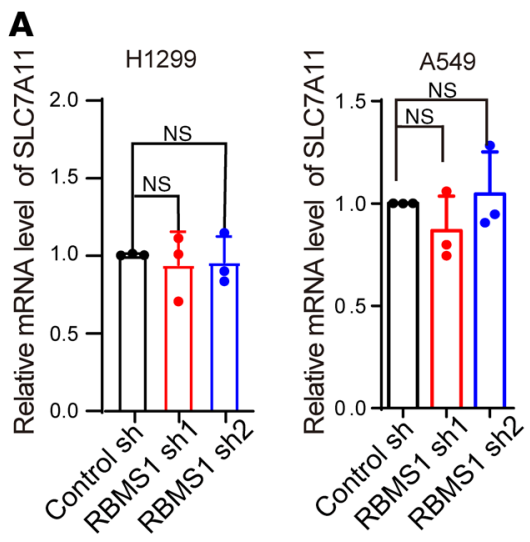

\section{SLC7A11-fluc-FL SLC7A11-fluc-FL RNA}

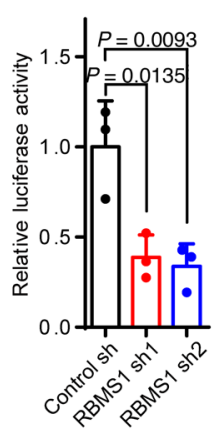

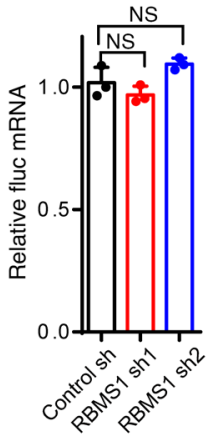

B

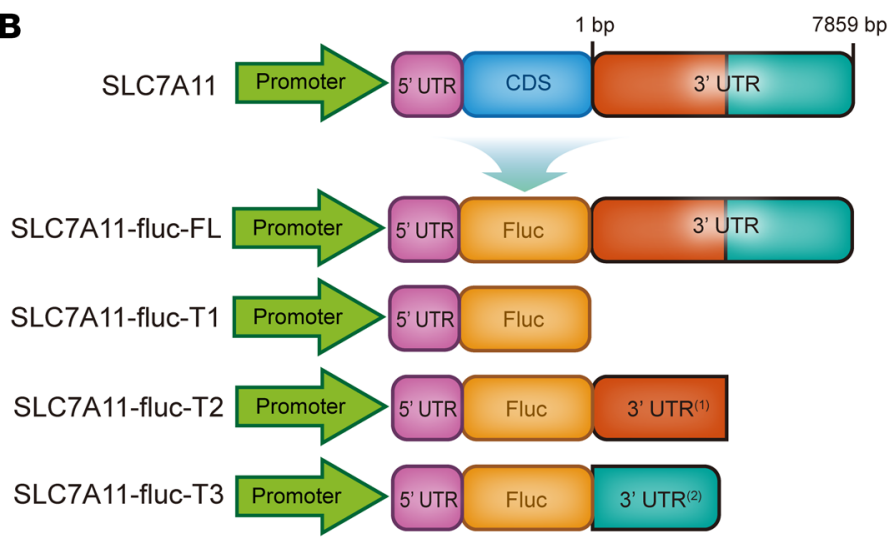

D SLC7A11-fluc-T1

SLC7A11-fluc-T2

SLC7A11-fluc-T3

E SLC7A11-fluc-T3
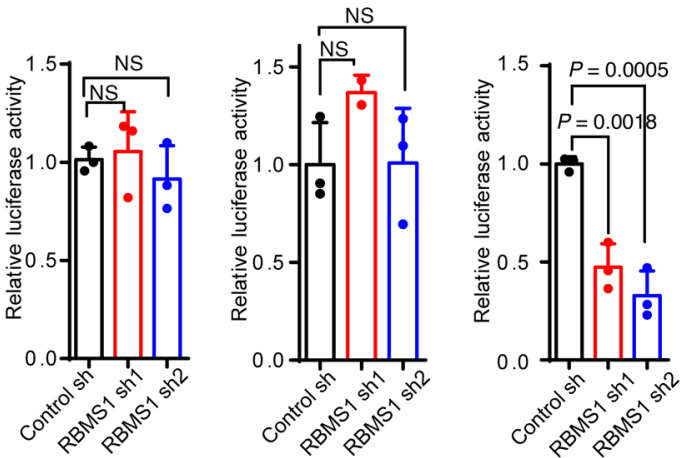

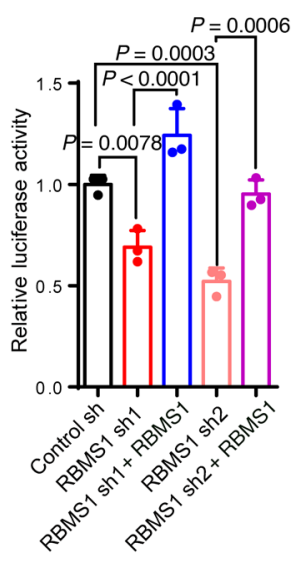

Figure 7. RBMS1 regulates SLC7A11 translation. (A) The mRNA levels of SLC7A11 in RBMS1-depleted H1299 and A549 cells were examined using qRTPCR. (B) Schematic of SLC7A11 luciferase reporter plasmids: SLC7A11-fluc-FL (promoter region, 5'-UTR, and 3'-UTR); SLC7A11-fluc-T1 (promoter region and 5'-UTR); SLC7A11-fluc-T2 (promoter region, 5'-UTR, and nt 1-3846 of 3'-UTR); and SLC7A11-fluc-T3 (promoter region, 5'-UTR, and nt 3827-7859 of 3'-UTR). (C) SLC7A11-fluc-FL was transiently transfected into RBMS1-depleted H1299 cells and the mRNA level of SLC7A11-fluc was examined using qRT-PCR. (D) SLC7A11-fluc-T1, SLC7A11-fluc-T2, and SLC7A11-fluc-T3 were transiently transfected into RBMS1-depleted H1299 cells. (E) SLC7A11-fluc-T3 was transiently transfected into RBMS1-depleted $\mathrm{H} 1299$ cells with or without reexpression of RBMS1. In C-E, the relative luciferase activities were determined by calculating the ratio of firefly luciferase activity over Renilla luciferase activity. Data represent mean \pm SEM, $n=3(\mathbf{A}$ and $\mathbf{C}-\mathbf{E})$ independent repeats. $P$ values were determined by 1-way ANOVA with Dunnett's multiple comparison test (A, C, and D) or 1-way ANOVA with Tukey's multiple comparison test (E).

SLC7A11 axis for lung cancer treatment, we performed a chemical screen to systematically identify inhibitors of RBMS1 that might promote ferroptosis using the US drug collection of compounds (https:// www.selleckchem.com/screening/fda-approved-drug-library.html), which contains 1280 drugs (38). We generated H1299 lung cancer cells with stable expression of EGFP-RBMS1 fusion protein, and then incubated these cells with distinct compounds (each at $2 \mu \mathrm{M}$ ) for 12 hours (Figure 12F). In total, 8 compounds were identified as reducing the fluorescence of EGFP-RBMS1 (Figure 12G). Nortriptyline hydrochloride (NTP), an antidepressant that inhibits the vascular Kv channel (39), was one of the top hits that suppressed the expression of RBMS1 (Figure 7O). We confirmed that NTP decreased the level of RBMS1 and significantly inhibited the activity of luciferase reporter SLC7A11-fluc-T3 in lung cancer cells (Figure 12H).

Importantly, NTP suppressed the expression of RBMS1 in IR-resistant A549 lung cancer cells and reduced the level of SLC7A11 (Figure 12I) without affecting the expression of other ferroptosis-related factors (Supplemental Figure 10F). In addition, NTP significantly promoted erastin-induced cell death, which could be rescued by the ferroptosis inhibitor Ferr-1, indicating that the treatment with NTP could also stimulate ferroptosis (Figure 12J and Supplemental Figure 10G). Consequently, the treatment with NTP sensitized the IR-resistant lung cancer cells to irradiation as compared with those cells treated with the vehicle (Figure 12K). Altogether, our data suggest that inhibition of RBMS1 by NTP sensitizes IR-resistant lung cancer cells to radiotherapy, providing a potentially novel and effective therapeutic agent for lung cancer patients by promoting ferroptosis.

\section{Discussion}

Ferroptosis can be regulated by a variety of factors and thus participate in regulating cancer progression. However, few studies have shown that RBPs play vital roles in ferroptosis regulation. For example, DAZAP1 was shown to bind the 3'-UTR of SLC7A11 mRNA and positively modulate its stability to inhibit ferroptosis (40). The RBP ZFP36 (also known as tristetraprolin, TTP) protects against ferroptosis by regulating the autophagy signaling pathway in hepatic stellate cells (41). Using an shRNA library against 


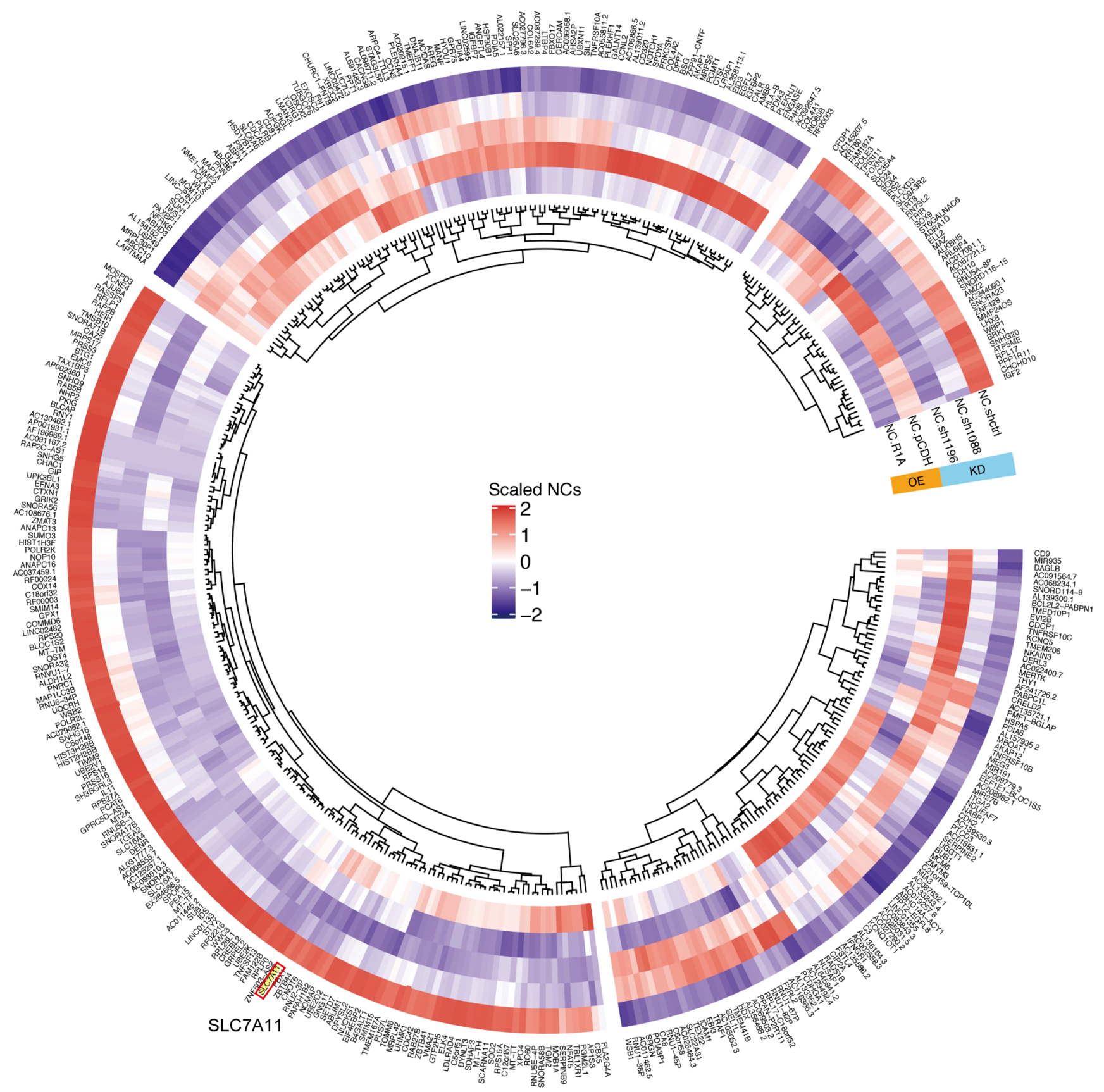

Figure 8. Ribo-seq analysis of RBMS1-depleted or -overexpressing cells. Ribo-seq assay was used to analyze RBMS1-depleted (knockdown, KD) and -overexpressing (OE) cells. Clustering heatmap of the differentially expressed genes was assessed with the Euclidean distance measurement in columns and $Z$ normalization in rows.

RBPs, we systematically showed that multiple RBPs could control ferroptosis, including RBMS1, SRSF9, TIAL1, PPIG, XRCC6, etc. Specifically, we revealed a model in which the RBP RBMS1 plays an important regulatory role in mediating ferroptosis, thereby controlling lung cancer progression. We demonstrated that RBMS1 binds to the eIF3d complex to bridge the $3^{\prime}$ - and $5^{\prime}$-UTR of SLC7A11, which in turn promotes the translation of SLC7A11. Subsequently, the increased SLC7A11 inhibits ferroptosis and confers radioresistance to lung cancer cells (Figure 12L). However, when RBMS1 is depleted, the SLC7A11 mRNA and eIF3d are released, resulting in the suppression of SLC7A11 translation. The reduced SLC7A11 promotes ferroptosis, thereby inhibiting lung cancer progression and sensitizing resistant cancer cells to radiotherapy (Figure 12L). Taken together, our data establish RBMS1 as a ferroptosis regulator with therapeutic potential and clinical value as a prognostic factor.

The RBP RBMS1 contains 2 RNA recognition motifs, and was previously reported to bind directly to the C-terminal portion of c-Myc, stimulating the cooperative transforming activity of c-Myc with Ras (42). In addition, as a posttranscriptional regula- 
A

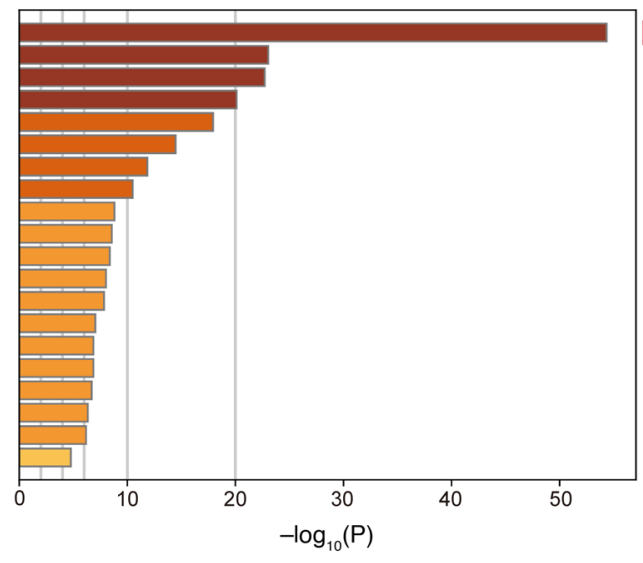

C

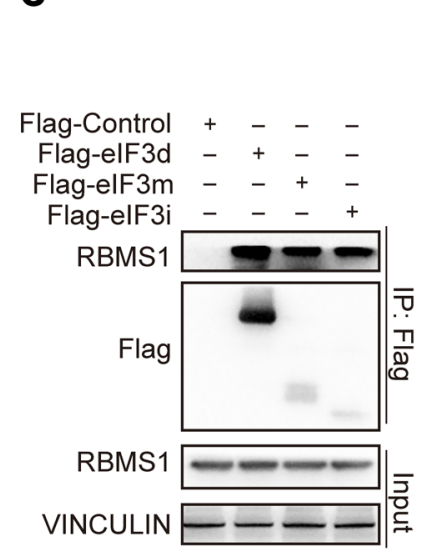

D

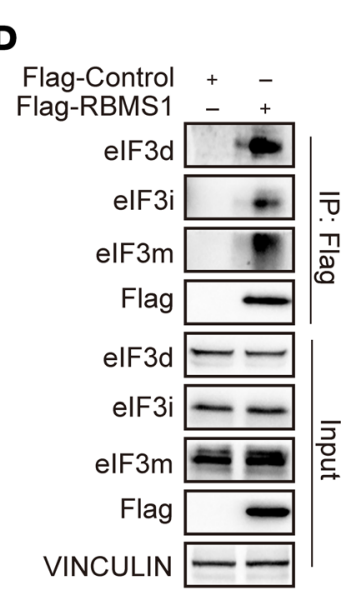

B Translation regulation mRNA processin mRNA binding
Ribonucleoprotein biogenesis Regulation of translation Translation initiation RNA localization

Ribonucleoprotein granule Nuclear-transcribed mRNA catabolic process ncRNA metabolic process Single-stranded RNA binding rRNA binding

Nuclear speckle

Ribonucleoprotein complex binding Regulation of RNA binding

Regulation of protein localization

Pseudouridine synthase activity Polysome

Unfolded protein binding Spliceosomal complex

E Flag-Control + - - + + - $\begin{array}{rllllllll}\text { Flag-elF3d } & - & - & - & + & - & - & - & + \\ \text { Flag-elF3i } & - & - & + & - & - & - & + & -\end{array}$

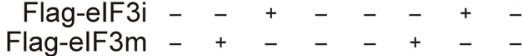

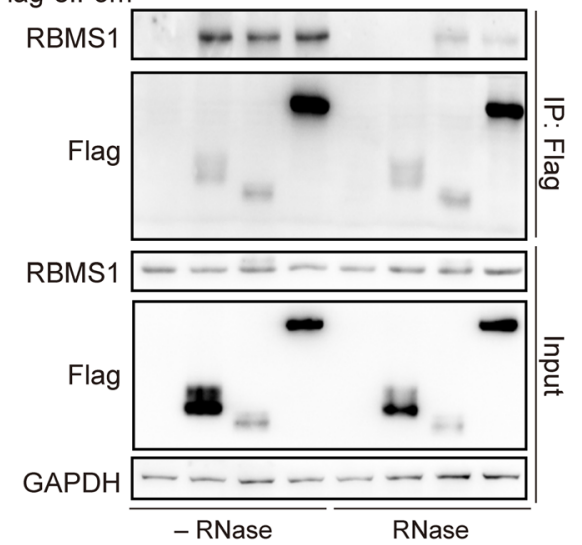

\section{$\mathbf{F}$}

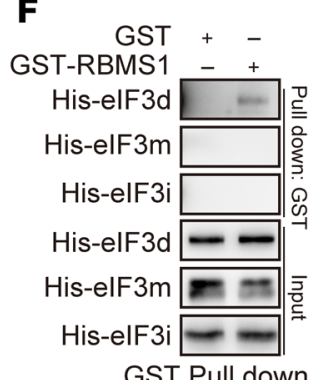

G
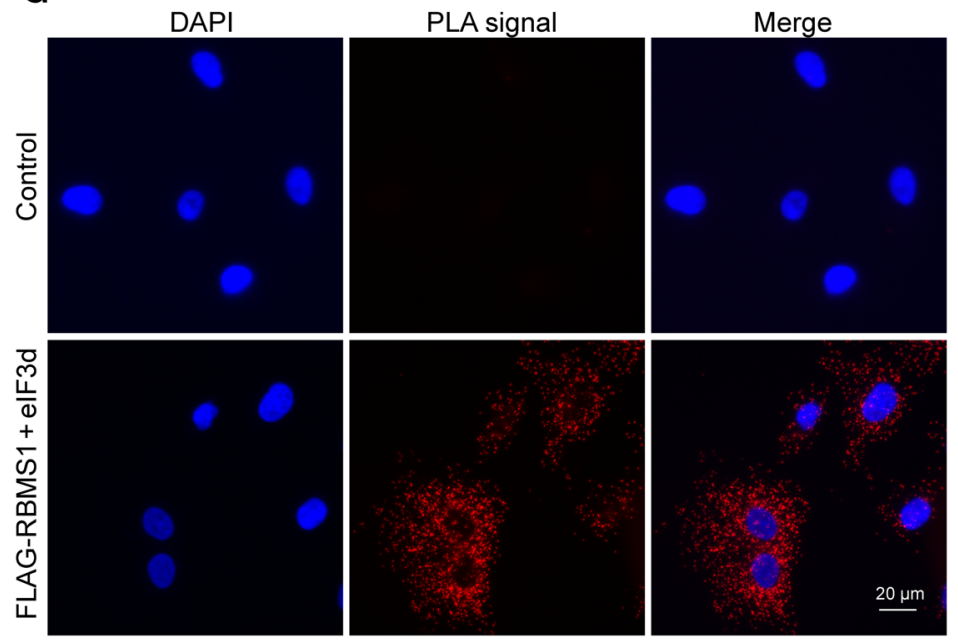

Figure 9. RBMS1 interacts with elF3d. (A) Gene ontology analysis of immunoprecipitation-coupled mass spectrometry (IP-MS) using cells expressing RBMS1. (B) Functional association network of genes from IP-MS were analyzed with the STRING database. (C and D) Immunoprecipitation was performed in $\mathrm{H} 1299$ cells expressing Flag-elF3d, Flag-elF3i, or Flag-elF3m (C), or H1299 cells expressing Flag-RBMS1 (D), and the precipitated complexes were analyzed. (E) H1299 cells expressing Flag-elF3d, Flag-elF3i, or Flag-elF3m were immunoprecipitated with or without RNase-A treatment, and the precipitated complexes were analyzed. (F) CST pull-down assays to analyze direct binding of recombinant GST-tagged human RBMS1 and His-tagged elF3d, elF3m, or elF3i. (G) PLA was performed to examine the interaction between RBMS1 and elF3d. PLA signals are shown in red and nuclei in blue. Scale bar: $20 \mu \mathrm{m}$. 
A

SLC7A11-fluc-T2 SLC7A11-fluc-T3
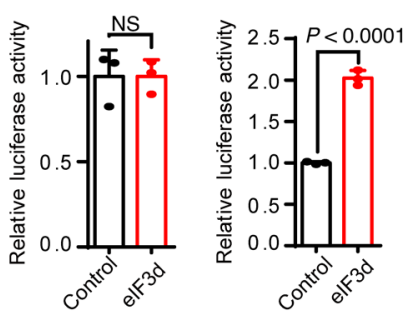

E $F$
B

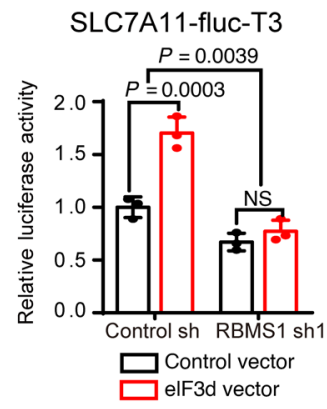

C

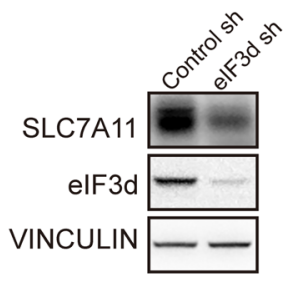

D

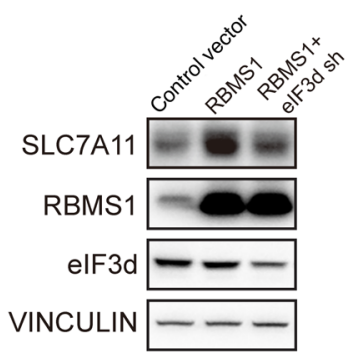

E

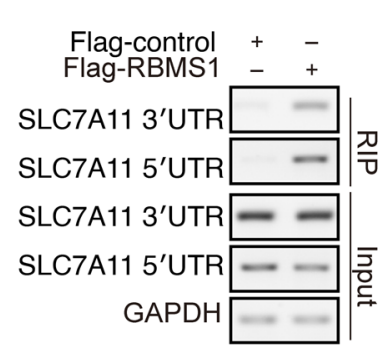

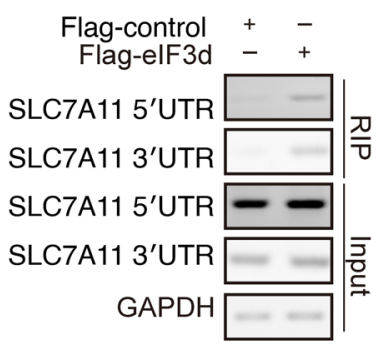

G

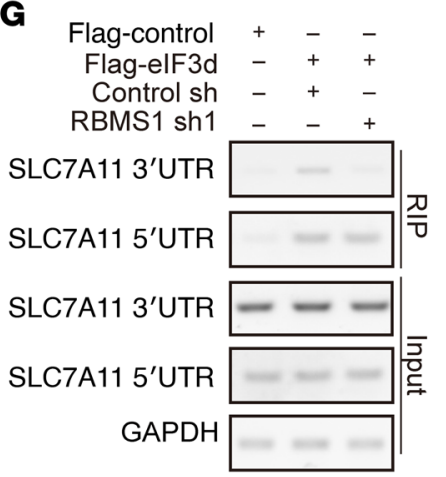

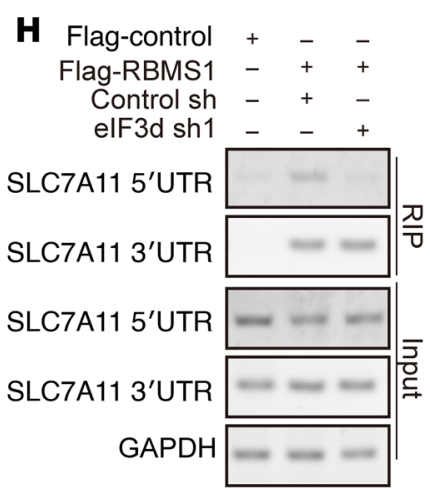

I

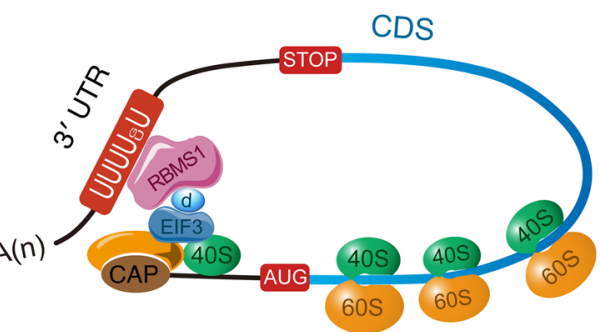

Figure 10. RBMS1 interacts with elF3d to bridge the 3'- and 5'-UTR of SLC7A11 to promote its translation. (A) SLC7A11-fluc-T2 and SLC7A11-fluc-T3 were transiently transfected into elF3d-overexpressing H1299 cells. (B) SLC7A11-fluc-T3 was transiently transfected into RBMS1-depleted H1299 cells with or without elF3d overexpression. For $\mathbf{A}$ and $\mathbf{B}$, data represent mean $\pm \mathrm{SEM}(n=3)$. $P$ values were calculated from unpaired $t$ test $(\mathbf{A})$ or 1 -way ANOVA with Tukey's multiple comparison test (B). (C) The level of SLC7A11 was examined in elF3d-depleted H1299 cells. (D) The levels of SLC7A11, RBMS1, and elF3d were examined in H1299 cells expressing RBMS1, with or without elF3d depletion. (E) Binding of SLC7A11 5'- and 3'-UTR with RBMS1 was examined by RNA-IP (RIP) in A549 cells expressing FLAG-RBMS1. (F) Binding of SLC7A11 5'- and 3'-UTR with elF3d was examined by RNA-IP in A549 cells expressing FLAG-elF3d. (G) Binding of SLC7A11 3'- or 5'-UTR with elF3d was examined by RNA-IP assay in A549 cells expressing FLAG-elF3d with or without RBMS1 depletion. (H) Binding of SLC7A11 5'- or 3'-UTR with RBMS1 was examined by RNA-IP assay in A549 cells expressing FLAG-RBMS1 with or without elF3d depletion. (I) Schematic of the model for how RBMS1 regulates the translation of SLC7A11.

tor of RNA stability, RBMS1 was shown to bind the 3'-UTR of target mRNAs, leading to increased mRNA stability (33). Functional study of RBMS1 revealed that RBMS1 silencing accompanies colorectal cancer progression in cell line and PDX models as well as clinical samples (33). However, our study demonstrated that the level of RBMS1 is markedly increased in lung cancer patient samples as compared with adjacent normal tissues, and loss of RBMS1 inhibits lung cancer progression in cultured cancer cells, xenograft mice, and genetically conditional knockout mice. The differential expression and distinct functions of RBMS1 might be determined by the tissue specificity and distinct localization of RBMS1, which are similar to that of PTEN $(43,44)$. Most importantly, we revealed that, instead of regulating mRNA stability, RBMS1 modulates the translation of SLC7A11, establishing a role for RBMS1 in cancer progression regulation.
As a critical regulator of ferroptosis, SLC7A11 could promote cancer progression partly through inhibiting ferroptosis. For example, a p53 mutant (3KR) retained its tumor-suppressive function partially by inhibiting SLC7A11 expression and inducing ferroptosis (8). SLC7A11 can also promote tumor development by directly interacting with ALOX12 and suppressing ALOX12-mediated ferroptosis in the absence of p53 (45). BAP1 inhibited cancer progression partly by triggering ferroptosis upon SLC7A11 repression (46). Meanwhile, the level of SLC7A11 could be regulated at distinct levels. Both p53 and BAP1 could regulate SLC7A11 at the transcriptional level $(8,46)$. The enzyme OTUB1 deubiquitinates and stabilizes SLC7A11 protein by posttranslational regulation (24). However, the translational regulation of SLC7A11 remains largely unknown. In our study, for the first time to our knowledge, we report that RBMS1 binds to the eIF3d complex to bridge the 3'- 
A

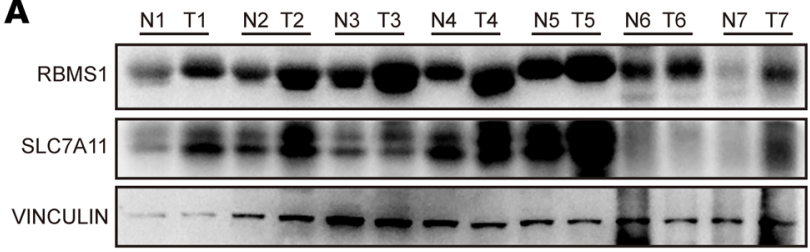

B

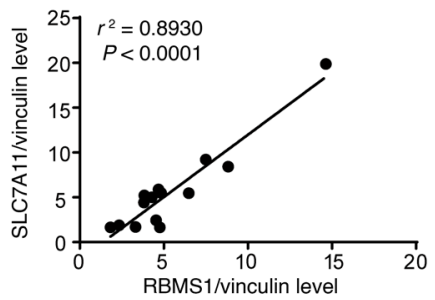

C

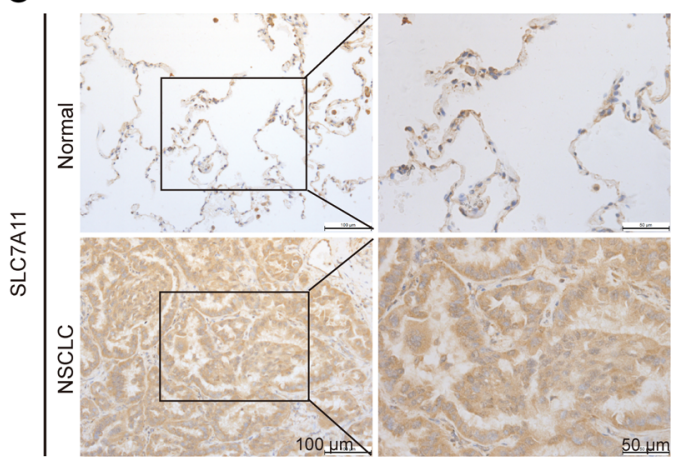

D Extra-strong positive Strong positive

Weak positive/negative

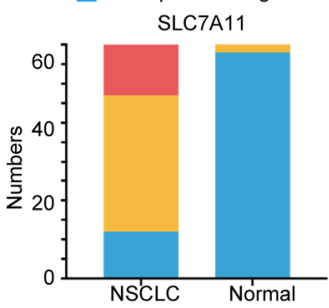

E

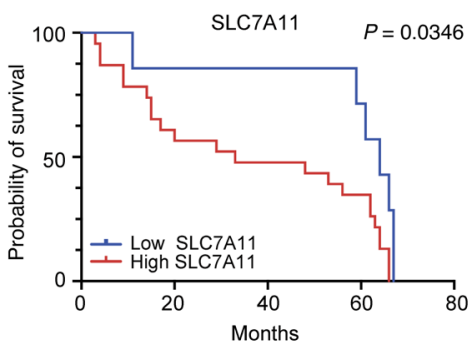

$\mathbf{F}$

$\mathrm{Kras}^{\mathrm{G12DNT}} / \mathrm{Rbms}^{\mathrm{WT}}$
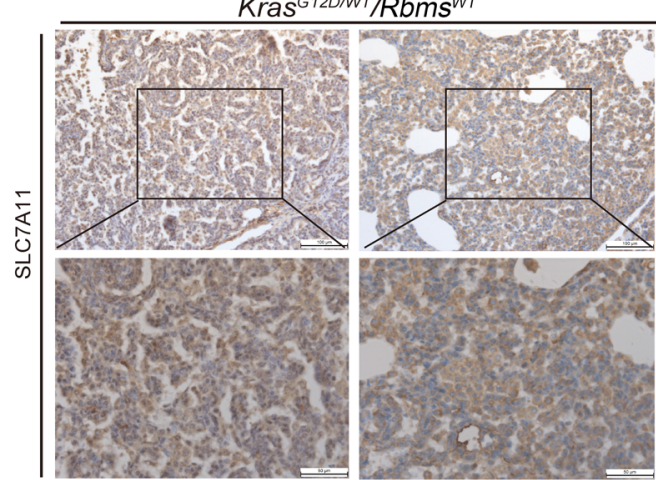

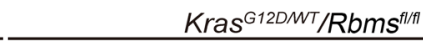
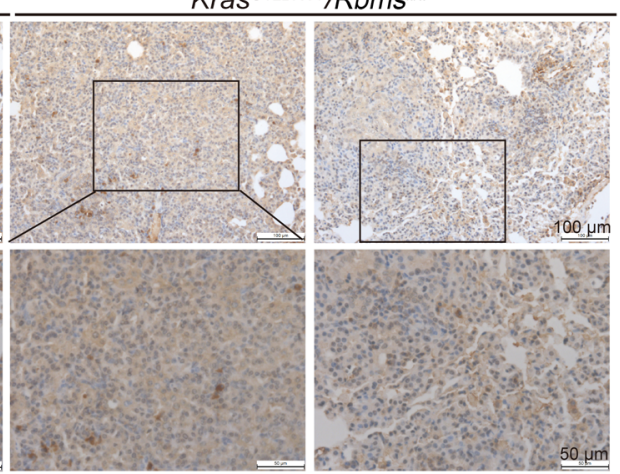

G

H
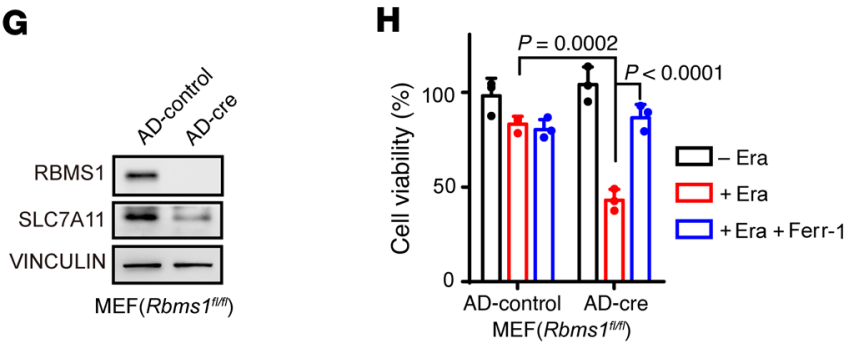

Figure 11. RBMS1 is positively correlated with SLC7A11 in clinical samples and mice. (A) RBMS1 and SLC7A11 levels of 7 paired lung cancer patient tumors (T) and adjacent tissues (N) were analyzed. (B) Correlation of SLC7A11 with RBMS1 levels was analyzed. (C) Representative images from immunohistochemical staining of SLC7A11 in lung cancers $(n=60)$ and normal tissues $(n=60)$. Scale bars: $100 \mu \mathrm{m}$ (left) and $50 \mu \mathrm{m}$ (right). (D) The relative immunohistochemical staining levels of SLC7A11 were quantified and classified into 3 grades. (E) Kaplan-Meier curve showing overall survival of lung cancer patients with high and low SLC7A11 expression. (F) Tumors were removed from Kras ${ }^{G 120 / W T} / R b m s 7^{W T}$ or CKO mice and subjected to immunohistochemical staining with an anti-SLC7A11 antibody. Scale bars: $100 \mu \mathrm{m}$ (top) and $50 \mu \mathrm{m}$ (bottom).(C) MEFs obtained from 14-day-old embryos of Rbmsfl/fl CKO mice were infected with AD-Cre to delete RBMS1 and levels of RBMS1 and SLC7A11 were examined. (H) Bar graphs showing cell viability of RBMS1-depleted MEFs treated with erastin, with or without Ferr-1. Data represent mean \pm SEM $(n=3)$. $P$ values were determined using 1-way-ANOVA with Tukey's multiple comparison test $(\mathbf{H})$.

and 5'-UTR of SLC7A11, which in turn promotes its translation. We revealed that loss of RBMS1 repressed the expression of SLC7A11 to stimulate ferroptosis.

In addition to RBMS1, we also identified some other RBPs that could regulate ferroptosis, including SRSF9, TIA1, XRCC6, etc. Several identified RBPs have been previously demonstrated to play critical roles in cancer progression. For instance, the RBP SRSF9 was reported to promote Wnt signaling-mediated tumorigenesis by enhancing $\beta$-catenin biosynthesis (47). Deregulated RNA binding of TIA1 promotes COX2 expression in colon cancer (48). XRCC6 directly interacts with Mcl1 to stabilize Mcl1, leading to suppression of apoptosis in lung cancer cells (49). However, 
A

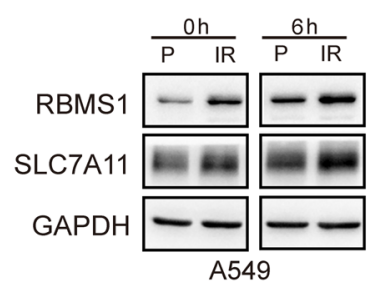

B

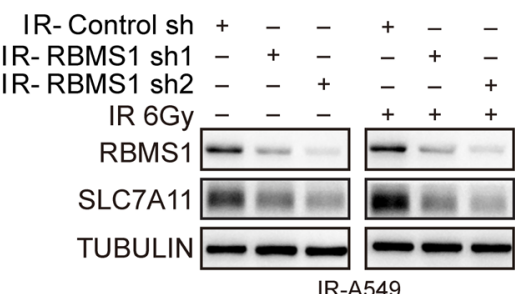

IR- Control sh + - - + -

IR-RBMS1 sh2 - $\quad+\quad+\quad-\quad+$

IR 6Gy -+-++
RBMS1 - - -+

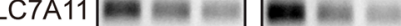

IR-A549

Lipid ROS level (FITC)

C

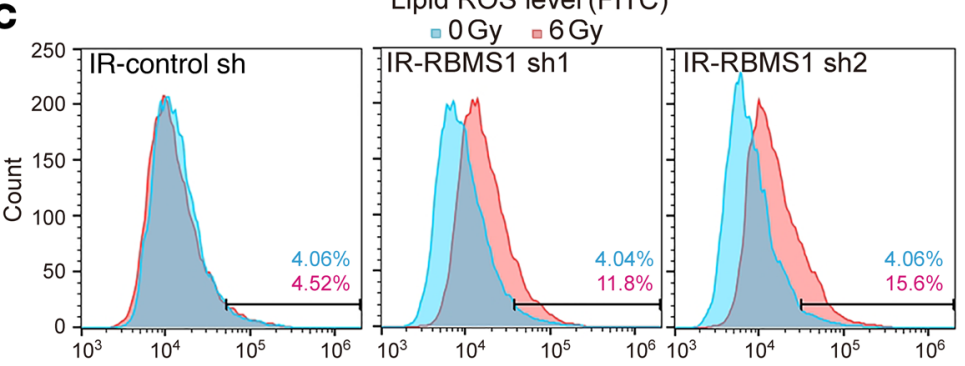

$\mathbf{F}$

U.S. drug collection screening
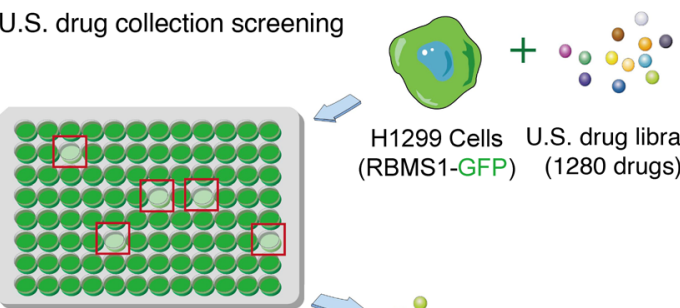

H1299 Cells U.S. drug library (RBMS1-GFP) (1280 drugs)

: Identified compounds
G

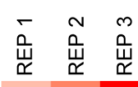

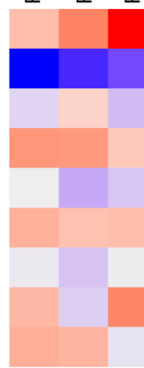

DMSO ZALEPLON SUCRALFATE MEQUINOL

NORTRIPTYLINE HYDROCHLORIDE NAFRONYL OXALATE AMINOGLUTETHIMIDE

ANTAZOLINE PHOSPHATE CEPHALOTHIN SODIUM

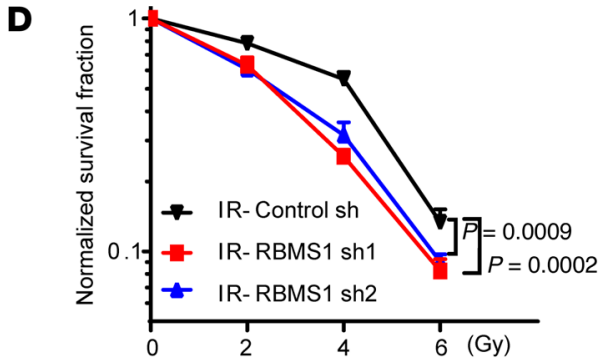

$\mathbf{E}$

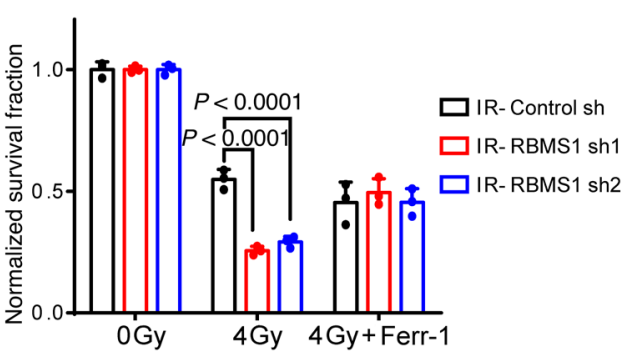

J

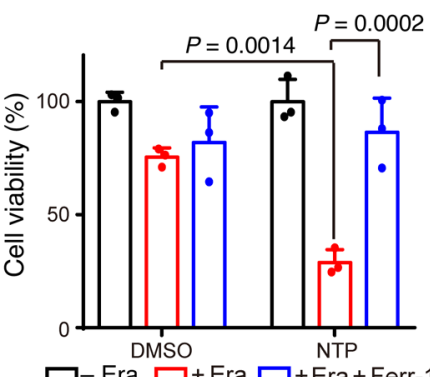

$\mathbf{L}$

$\mathbf{K}$ ロ-Era $\square+E r a \square+E r a+F e r r-1$
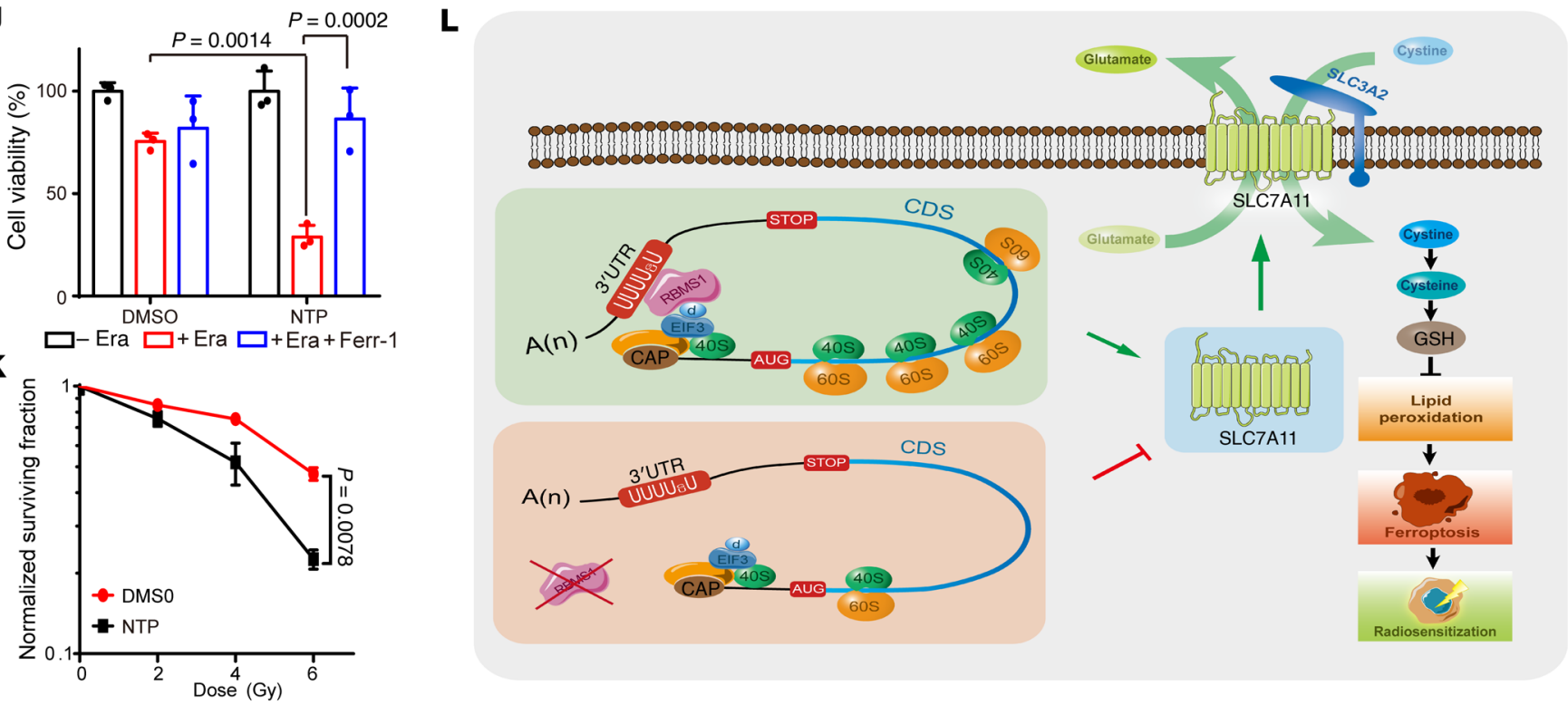

Figure 12. RBMS1 regulates lung cancer cell radioresistance through SLC7A11. (A and B) RBMS1 and SLC7A11 levels in irradiation-resistant (IR-resistant) A549 cells with or without irradiation (A) and with or without RBMS1 depletion (B) were measured. (C) Lipid peroxidation was measured by flow cytometry after C11BODIPY staining in RBMS1-depleted IR-resistant A549 cells with or without irradiation. (D and E) Clonogenic survival was examined in RBMS1-depleted IR-resistant $A 549$ cells treated with different radiation dosages (D), or irradiation with and without Ferr-1 (E). (F) H1299 cells expressing pECFP-RBMS1 were treated with 1280 compounds and fluorescence intensities were examined. (G) Heatmap depicting the GFP intensity in A549 cells treated with 8 identified compounds. (H) IR-resistant A549 cells were transfected with SLC7A11-fluc-T3 and treated with nortriptyline hydrochloride (NTP). The luciferase activities and RBMS1 levels were examined. (I) RBMS1 and SLC7A11 levels in NTP-treated IR-resistant A549 cells were examined. (J) Bar graphs showing viability of IR-resistant A549 cells treated with NTP or erastin, with or without Ferr-1. (K) Clonogenic survival was examined in NTP-treated IR-resistant A549 cells with different radiation dosages. (L) The schematic of how RBMS1 regulates SLC7A11 translation and ferroptosis in lung cancer. Data represent mean \pm SEM $(n=3)$. $P$ values were determined using 1-way ANOVA with Tukey's multiple comparison test (E and J), 1-way repeated measures ANOVA ( $\mathbf{D}$ and $\mathbf{K})$, or unpaired $t$ test (H). 
it remains largely elusive whether these RBPs could participate in cancer progression regulation through controlling ferroptosis. Therefore, we will further mechanistically study the functions of such RBPs in mediating ferroptosis, providing potential targets for cancer therapeutics in the future.

\section{Methods}

Reagents. Erastin was obtained from AbMole (M2679). TBH and Ferr-1 were obtained from Sigma-Aldrich (458139 and SML0583). Z-VADFMK (S7023), 3-MA (S2767), liproxstatin-1 (S7699), NAC (S1623), and the US drug collection were obtained from Selleck.

Cell culture. A549, NCI-H1299, and HEK293T cells were purchased from the American Type Culture Collection (ATCC). All of the cells were cultured under standard culture conditions $\left(37^{\circ} \mathrm{C}, 5 \%\right.$ $\mathrm{CO}_{2}$ ) in culture medium recommended by the ATCC. To generate RBMS1 overexpression or stable-knockdown cell lines, HEK293T cells were transfected with either pCDH-Flag-RBMS1 or pLKO.1 RBMS1 constructs, together with pPAX2 and pMD2 lentiviral packaging systems using LipoPlus reagent (Sage) according to the manufacturer's instructions. Viruses were collected 72 hours after transfection and then used to infect A549 and NCI-H1299 cells with Polybrene ( $8 \mu \mathrm{g} / \mathrm{mL}$, Sigma-Aldrich) for 24 hours. Then, stably integrated cells were selected with $5 \mu \mathrm{g} / \mathrm{mL}$ puromycin for 5 days. To generate the doxorubicin-inducible RBMS1-knockdown stable cell line, pLKO-Tet-On-RBMS1-shRNA was transfected into HEK293T cells together with pPAX2 and pMD2 lentiviral packaging systems using LipoPlus reagent according to the manufacturer's instructions. Viruses were harvested 72 hours after transfection. A549 and NCI-H1299 cells were infected for 24 hours followed by puromycin $(5 \mu \mathrm{g} / \mathrm{mL})$ selection. For CRISPR knockout cell construction, sgRNA was introduced into the lentiCRISPRv2 vector and packaged in lentivirus, and infected cells were screened with puromycin to obtain the stable cell line. In the experiments involving cystine depletion, fresh DMEM was used as the control, and the conditioned DMEM (21013024, Gibco) supplemented with $1 \mathrm{mM}$ sodium pyruvate, 0.2 $\mathrm{mM}$ L-methionine, $4 \mathrm{mM} \mathrm{L}$-glutamate, and 10\% nondialyzed FBS was used as low-cystine treatment.

Plasmid construction. To generate the mammalian expression plasmids pCDH-Flag-RBMS1, pCDH-Flag-SLC7A11, and pCDH-FlageIF3d, human RBMS1, SLC7A11, and eIF3d cDNAs were PCR amplified and then cloned into the lentivirus vector pCDH-CMV-MCSEF1-Puro with N-terminal Flag tag with restriction enzymes NheI and NotI. shRNAs targeting RBMS1 were cloned into the pLKO.1 plasmid and pLKO-Tet-On inducible vector. For bacterial protein expression of human eIF3d, eIF3i, and eIF3m that express N-terminal His-fused proteins, the eIF3d, eIF3i, or eIF3m cDNA was inserted into pET$28 \mathrm{a}(+)$ with EcoRI-NotI sites. For expression of recombinant RBMS1 proteins, RBMS1 cDNA was cloned into the pGEX4T1-GST vector with EcoRI and NotI sites. To generate SLC7A11 luciferase reporter, the DNA fragment of the human SLC7A11 gene promoter and 5'-UTR $(-679 /+280)$ was amplified by PCR and sub-cloned into the NheI-BglII sites of the pGL3 enhancer vector (SLC7A11-fluc-T1). All constructs were confirmed by DNA sequencing. Primers for PCR amplification, gRNAs, and shRNAs are listed in Supplemental Table 2.

Cell viability and cell death assays. For viability assays, cells were seeded in 96-well plates and treated with drugs for an appropriate time on the next day. Then, medium with drugs was removed and replacedwith fresh medium containing $10 \%$ Cell Counting Kit-8 (CCK8) reagent (APExBIO). After incubation for 2 hours at $37^{\circ} \mathrm{C}$, the plate was analyzed using an ELISA reader (DNM-9602, Perlong) and absorbance of the wells was measured at $450 \mathrm{~nm}$. In order to detect cell death, cells were seeded in 12-well plates and treated with drugs for an appropriate time to induce cell death on the next day. Cells were digested with trypsin to obtain cell pellets, which were suspended with PBS. Cells were stained by mixing $100 \mu \mathrm{L}$ cell suspension and $200 \mu \mathrm{L} 0.02 \%$ trypan blue and incubating for 1 minutes, and the cells were counted with a Countess II FL automated cell counter (Thermo Fisher Scientific) to obtain the proportion of dead cells. Alternatively, $500 \mu \mathrm{L}$ of cells in PBS were mixed with $5 \mu \mathrm{L}$ propidium iodide (KGA214, KeyGEN BioTECH). After staining for 15 minutes, the proportion of dead cells was analyzed by an Accuri C6 Plus personal flow cytometer (BD Biosciences).

GSH assay. GSH levels were measured using a GSH-Glo Glutathione Assay kit (Promega). In brief, cells were seeded at 6000 cells per well in 96-well plates. The medium was removed on the next day, and $100 \mu \mathrm{L} 1 \times$ GSH-GLO Reagent was added to each well and incubated for 30 minutes at room temperature. Then, 100 $\mu \mathrm{L}$ reconstituted Luciferin Detection Reagent was added to every well, mixed gently, and incubated at room temperature for 15 minutes. Luminescence was detected by a multifunctional plate reader (Enspire 2300, PerkinElmer).

Lipid peroxidation assay. Cells were seeded in 6-well plates and treated with drugs for an appropriate time on the next day, and then treated with $5 \mu \mathrm{M}$ BODIPY 581/591 C11 (D3861, Thermo Fisher Scientific) and incubated at $37^{\circ} \mathrm{C}$ for 30 minutes. The cells were then washed twice with $1 \mathrm{~mL} 1 \times$ PBS, digested with trypsin to obtain cell pellets, and suspended with $500 \mu \mathrm{L}$ PBS. A BD Accuri C6 Plus personal flow cytometer was used to analyze changes in fluorescence.

${ }^{13} \mathrm{C}$-cystine labeling experiments. H1299 cells with stable RBMS1 knockdown or pLKO.1 control plasmid were plated on 60-mm dishes for 12 hours. Cells were incubated in fresh DMEM (21013024, Gibco) with $1 \mathrm{mM}$ sodium pyruvate, $0.2 \mathrm{mM} \mathrm{L}$-methionine, and $4 \mathrm{mM} \mathrm{L}$-glutamate for 4 hours. The medium was then replaced with fresh DMEM (without cystine) supplemented with $0.2 \mathrm{mM}{ }^{13} \mathrm{C}$-labeled L-cystine $\left(3,3^{\prime}-{ }^{13} \mathrm{C} 2\right)$ (Cambridge Isotope Laboratories, CLM-520-PK) for 4 hours, after which the cells were quickly washed 3 times with PBS to remove contamination from the media. The PBS was thoroughly aspirated and cells were immediately frozen in liquid nitrogen and then stored at $-80^{\circ} \mathrm{C}$ for GC-MS analysis. Protein concentrations were determined by processing parallel 6-well plates and were used to normalize metabolite fractions.

Light microscopy and immunofluorescence microscopy. For light microscopy, cells were cultured in 6-well plates and treated with reagents as indicated. Phase-contrast images were captured using a Leica microscope equipped with a $10 \times$ phase-contrast objective. For immunofluorescence microscopy, cells with stable transfection of pCDH-FLAG-RBMS1 plasmid seeded on glass slides were cultured for 24 hours. And after removing medium with PBS, cells were fixed with $4 \%$ paraformaldehyde for 20 minutes followed by washing with PBS 3 times for 5 minutes each, and then blocked with 3\% BSA in PBS for 10 minutes. The cells were then incubated with primary antibodies anti-FLAG (Sigma-Aldrich, F3165, 1:100) and anti-calnexin (Cell Signaling Technology, 2679s, 1:100) overnight at $4^{\circ} \mathrm{C}$ and then 
washed with PBS 3 times for 5 minutes each. Next, fluorescently labeled secondary antibodies were incubated with the cells for 1 hour at room temperature and removed by washing 3 times with PBS for 5 minutes each and then nuclei were labeled with DAPI. Finally, fluorescently labeled target proteins were detected using a Leica Mi8 microscope (oil immersion objective).

TEM. For ultrastructural analysis of mitochondria, TEM was used. Stably RBMS1-depleted or control H1299 cells were treated with or without $5 \mu \mathrm{M}$ erastin, and fixed with $2.5 \%$ glutaraldehyde in $0.1 \mathrm{M}$ PBS (pH 7.4) at $4^{\circ} \mathrm{C}$ for 2.5 hours, washed 3 times with $0.1 \mathrm{M}$ PBS, and postfixed in $1 \% \mathrm{OsO}_{4}$ for 2 hours at $4^{\circ} \mathrm{C}$. The samples were then dehydrated through an ethanol gradient and subsequently embedded in Spurr's resin. Ultrathin sections were then collected and stained with either uranyl acetate or lead citrate and examined using a JEOL 1200EX transmission electron microscope.

SIM. Cells were incubated with $2 \mu \mathrm{M}$ Rho123 (J\&K Scientific, 62669-70-9) at $37^{\circ} \mathrm{C}$ and $5 \% \mathrm{CO}_{2}$ for 2 hours to stain the mitochondria. Subsequently, cells were washed twice with $1 \mathrm{~mL}$ RPMI-1640 medium containing 10\% FBS and imaged by SIM (Nikon N-SIM Ti-2E). Super-resolution images were obtained with a Nikon N-STORM/SIM 5.0 Super-Resolution Microscope System with a motorized inverted ECLIPSE Ti2-E microscope, a 100×/NA 1.49 oil immersion TIRF objective lens (CFI HP), LU-NV series laser unit, and an ORCA-Flash 4.0 SCMOS camera (Hamamatsu Photonics K.K.).

RNA isolation and qRT-PCR. RNA was extracted from cells or RNAIP samples using TRIzol reagent (Invitrogen) according to the manufacturer's instructions. Genomic DNAs were removed by 30-minute DNase I (Promega M6101) treatment at $37^{\circ} \mathrm{C}$. An equal volume of Stop Solution was added to terminate the reaction, which was then incubated at $65^{\circ} \mathrm{C}$ for 10 minutes to inactive the DNase. Total RNA $(2 \mu \mathrm{g})$ was then reverse transcribed with a PrimeScript RT reagent kit (Takara) with random primers. We performed real-time PCR using the Maxima SYBR Green qPCR Master Mix (Thermo Fisher Scientific) and a 7500 real-time PCR system (Life Technologies) according to the manufacturer's instructions.

Statistics. Data are presented as mean \pm SEM. Statistical significance was determined by 2-tailed Student's $t$ test, 1-way ANOVA with
Dunnett's multiple comparison test, 1-way repeated measures ANOVA, or 1-way ANOVA with Tukey's multiple comparison test, as appropriate.

Study approval. The Institutional Animal Care and Use Committee of the Dalian Medical University approved the use of animal models in this study.

All human tumor tissues were obtained with written informed consent from patients or their guardians prior to participation in the study. The Institutional Review Board of the Dalian Medical University approved use of the tumor specimens in this study.

Data availability. Ribo-seq data in this study have been deposited in NCBI's Gene Expression Omnibus (GEO GSE171640). The authors declare that all the data supporting the findings of this study are available within the article and its supplemental files.

\section{Author contributions}

YW conceived the project and designed the experiments. WZ, YS, LB, LZ, Y Yang, QZ, YQ, WG, WH, LW, HC, QQ, J Zhang, Y Yin, and $\mathrm{CP}$ designed and performed most of the experiments, whereas CC, DC, SF, HLP, ZX, J Zhao, SZ, XL, HL, and QL performed data analysis. YW provided funds. YW and XL wrote the manuscript.

\section{Acknowledgments}

This work was supported by the National Natural Science Foundation of China (grants 81830088, 81422038, 91540110, and 31471235 to YW; 81872247 and 31400726 to WZ); the Liaoning Revitalization Talents Program (grant XLYC1802067 to YW); the Department of Science and Technology of Liaoning Province (grant 2021JH6/10500160 to YW); the Newton Advanced Fellowship from the Academy of Medical Sciences in the UK (grant JXR11831 to YW); the Dalian High Level Talents Renovation Supporting Program (grant 2019RQ097 to WZ); and the Youth Innovation Promotion Association CAS (grant 2019267 to Y Yang).

Address correspondence to: Yang Wang, Institute of Cancer Stem Cells and Second Affiliated Hospital, Dalian Medical University, Dalian, China 116044. Phone: 86.411.86110531; Email: yangwang@dmu.edu.cn.
1. Dixon SJ, et al. Ferroptosis: an iron-dependent form of nonapoptotic cell death. Cell. 2012;149(5):1060-1072.

2. Yang WS, Stockwell BR. Ferroptosis: death by lipid peroxidation. Trends Cell Biol. 2016;26(3):165-176.

3. Xie Y, et al. Ferroptosis: process and function. Cell Death Differ. 2016;23(3):369-379.

4. Tang D, et al. Ferroptosis: molecular mechanisms and health implications. Cell Res. 2021;31(2):107-125.

5. Chen X, et al. Broadening horizons: the role of ferroptosis in cancer. Nat Rev Clin Oncol. 2021;18(5):280-296.

6. Zhu S, et al. HSPA5 regulates ferroptotic cell death in cancer cells. Cancer Res. 2017;77(8):2064-2077.

7. Zhang Y, et al. BAP1 links metabolic regulation of ferroptosis to tumour suppression. Nat Cell Biol. 2018;20(10):1181-1192.

8. Jiang L, et al. Ferroptosis as a p53-mediated activity during tumour suppression. Nature.
2015;520(7545):57-62.

9. Jennis M, et al. An African-specific polymorphism in the TP53 gene impairs p53 tumor suppressor function in a mouse model. Genes Dev. 2016;30(8):918-930.

10. Roh JL, et al. Induction of ferroptotic cell death for overcoming cisplatin resistance of head and neck cancer. Cancer Lett. 2016;381(1):96-103.

11. Tsoi J, et al. Multi-stage differentiation defines melanoma subtypes with differential vulnerability to drug-induced iron-dependent oxidative stress. Cancer Cell. 2018;33(5):890-904.

12. Viswanathan VS, et al. Dependency of a therapy-resistant state of cancer cells on a lipid peroxidase pathway. Nature. 2017;547(7664):453-457.

13. Hangauer MJ, et al. Drug-tolerant persister cancer cells are vulnerable to GPX4 inhibition. Nature. 2017;551(7679):247-250.

14. Wang W, et al. CD8 ${ }^{+} \mathrm{T}$ cells regulate tumour ferroptosis during cancer immunotherapy. Nature. 2019;569(7755):270-274.

15. Lei $\mathrm{G}$, et al. The role of ferroptosis in ionizing radiation-induced cell death and tumor suppression. Cell Res. 2020;30(2):146-162.

16. Lang X, et al. Radiotherapy and immunotherapy promote tumoral lipid oxidation and ferroptosis via synergistic repression of SLC7A11. Cancer Discov. 2019;9(12):1673-1685.

17. Koppula P, et al. Cystine transporter SLC7A11/xCT in cancer: ferroptosis, nutrient dependency, and cancer therapy. Protein Cell. 2020;12(8):599-620.

18. Koppula P, et al. Amino acid transporter SLC7A11/xCT at the crossroads of regulating redox homeostasis and nutrient dependency of cancer. Cancer Commun (Lond). 2018;38(1):12.

19. Yang WS, et al. Regulation of ferroptotic cancer cell death by GPX4. Cell. 2014;156(1-2):317-331.

20. Friedmann Angeli JP, et al. Inactivation of the ferroptosis regulator $\mathrm{Gpx} 4$ triggers acute renal failure in mice. Nat Cell Biol. 2014;16(12):1180-1191.

21. Conrad M, Sato H. The oxidative stress-inducible cystine/glutamate antiporter, system $\mathrm{x}(\mathrm{c})$ (-) : cystine supplier and beyond. Amino Acids. 
2012;42(1):231-246.

22. Rojo de la Vega M, et al. NRF2 and the hallmarks of cancer. Cancer Cell. 2018;34(1):21-43.

23. Badeaux AI, Shi Y. Emerging roles for chromatin as a signal integration and storage platform. Nat Rev Mol Cell Biol. 2013;14(4):211-224.

24. Liu T, et al. The deubiquitylase OTUB1 mediates ferroptosis via stabilization of SLC7A11. Cancer Res. 2019;79(8):1913-1924.

25. Ishimoto $\mathrm{T}$, et al. CD44 variant regulates redox status in cancer cells by stabilizing the $\mathrm{xCT}$ subunit of system xc(-) and thereby promotes tumor growth. Cancer Cell. 2011;19(3):387-400.

26. Li F, et al. HnRNP-F regulates EMT in bladder cancer by mediating the stabilization of Snail1 mRNA by binding to its 3' UTR. EBioMedicine. 2019;45:208-219.

27. Wang Y, et al. The splicing factor RBM4 controls apoptosis, proliferation, and migration to suppress tumor progression. Cancer Cell. 2014;26(3):374-389.

28. Chen TM, et al. hnRNPM induces translation switch under hypoxia to promote colon cancer development. EBioMedicine. 2019;41:299-309.

29. Boise LH, et al. bcl-x, a bcl-2-related gene that functions as a dominant regulator of apoptotic cell death. Cell. 1993;74(4):597-608.

30. Warzecha CC, et al. ESRP1 and ESRP2 are epithelial cell-type-specific regulators of FGFR2 splicing. Mol Cell. 2009;33(5):591-601.

31. Zhou L, et al. Oncogene SRSF3 suppresses autophagy via inhibiting BECN1 expression. Biochem
Biophys Res Commun. 2019;509(4):966-972.

32. Kimura K, et al. c-Myc gene single-strand binding protein-1, MSSP-1, suppresses transcription of alpha-smooth muscle actin gene in chicken visceral smooth muscle cells. Nucleic Acids Res. 1998;26(10):2420-2425.

33. Yu J, et al. RBMS1 suppresses colon cancer metastasis through targeted stabilization of Its mRNA regulon. Cancer Discov. 2020;10(9):1410-1423.

34. Van Nostrand EL, et al. A large-scale binding and functional map of human RNA-binding proteins. Nature. 2020;583(7818):711-719.

35. Mao C, et al. DHODH-mediated ferroptosis defence is a targetable vulnerability in cancer. Nature. 2021;593(7860):586-590.

36. Lee AS, et al. eIF3d is an mRNA cap-binding protein that is required for specialized translation initiation. Nature. 2016;536(7614):96-99.

37. Shi Y, et al. Nuclear EGFR-PKM2 axis induces cancer stem cell-like characteristics in irradiation-resistant cells. Cancer Lett. 2018;422:81-93.

38. Cui B, et al. Stress-induced epinephrine enhances lactate dehydrogenase A and promotes breast cancer stem-like cells. J Clin Invest. 2019;129(3):1030-1046.

39. Shin SE, et al. Nortriptyline, a tricyclic antidepressant, inhibits voltage-dependent $\mathrm{K}^{+}$channels in coronary arterial smooth muscle cells. Korean J Physiol Pharmacol. 2017;21(2):225-232.

40. Wang Q, et al. RNA binding protein DAZAP1 promotes HCC progression and regulates ferroptosis by interacting with SLC7A11 mRNA. Exp Cell Res.
2021;399(1):112453.

41. Zhang Z, et al. RNA-binding protein ZFP36/TTP protects against ferroptosis by regulating autophagy signaling pathway in hepatic stellate cells. Autophagy. 2020;16(8):1482-1505.

42. Niki T, et al. MSSP promotes ras/myc cooperative cell transforming activity by binding to c-Myc. Genes Cells. 2000;5(2):127-141.

43. Kim SK, et al. Alterations of PTEN/MMAC1, a candidate tumor suppressor gene, and its homologue, PTH2, in small cell lung cancer cell lines. Oncogene. 1998;16(1):89-93.

44. Xie P, et al. Neddylation of PTEN regulates its nuclear import and promotes tumor development. Cell Res. 2021;31(3):291-311.

45. Chu B, et al. ALOX12 is required for p53mediated tumour suppression through a distinct ferroptosis pathway. Nat Cell Biol. 2019;21(5):579-591.

46. Zhang Y, et al. BAP1 suppresses tumor development by inducing ferroptosis upon SLC7A11 repression. Mol Cell Oncol. 2019;6(1):1536845.

47. Fu Y, et al. SRSF1 and SRSF9 RNA binding proteins promote Wnt signalling-mediated tumorigenesis by enhancing $\beta$-catenin biosynthesis. EMBO Mol Med. 2013;5(5):737-750.

48. Dixon DA, et al. Regulation of cyclooxygenase-2 expression by the translational silencer TIA-1. JExp Med. 2003;198(3):475-481.

49. Wang B, et al. Role of Ku70 in deubiquitination of Mcl-1 and suppression of apoptosis. Cell Death Differ. 2014;21(7):1160-1169. 